\title{
Design and Analysis of High Speed Capacitive Pipeline DACs
}

\author{
Quoc-Tai Duong, Jerzy Dabrowski and Atila Alvandpour
}

\section{Linköping University Post Print}

\section{Tweet}

N.B.: When citing this work, cite the original article.

The original publication is available at www.springerlink.com:

Quoc-Tai Duong, Jerzy Dabrowski and Atila Alvandpour, Design and Analysis of High Speed Capacitive Pipeline DACs, 2014, Analog Integrated Circuits and Signal Processing, (80), 3, 359-374.

http://dx.doi.org/10.1007/s10470-014-0350-9

Copyright: Springer Verlag (Germany)

http://www.springerlink.com/?MUD=MP

Postprint available at: Linköping University Electronic Press

http://urn.kb.se/resolve?urn=urn:nbn:se:liu:diva-105516 


\section{Design and Analysis of High Speed Capacitive Pipeline DACs}

\begin{abstract}
Design of a high speed capacitive digital-to-analog converter (SC DAC) is presented for $65 \mathrm{~nm}$ CMOS technology. SC pipeline architecture is used followed by an output driver. For GHz frequency operation with output voltage swing suitable for wireless applications $\left(300 \mathrm{mV}_{\mathrm{pp}}\right)$ the DAC performance is shown to be limited by the clock feed-through and settling effects in the $\mathrm{SC}$ array rather than by the capacitor mismatch or $\mathrm{kT} / \mathrm{C}$ noise, which appear negligible in this application. While it is possible to design a highly linear output driver with $\mathrm{HD3}<-70 \mathrm{~dB}$ and HD2 $<-90 \mathrm{~dB}$ over 0.5-5 GHz band as we show, the maximum SFDR of the SC DAC is $45 \mathrm{~dB}$ with 8-bit resolution and Nyquist sampling of $3 \mathrm{GHz}$. The capacitor array is designed based on the DAC design area defined in terms of the switch size and unit capacitance value. A tradeoff between the DAC bandwidth and resolution accompanied by SFDR is demonstrated. High linearity of the output driver is attained by a combination of two techniques, the derivative superposition (DS) and resistive source degeneration. In simulations the complete DAC achieves SFDR of $45 \mathrm{~dB}$ with 8-bit resolution for signal bandwidth $1.36 \mathrm{GHz}$ with Nyquist sampling. With 6-bit and 5.5 GHz bandwidth $33 \mathrm{~dB}$ SFDR is attained. The total power consumption of the SC DAC is $90 \mathrm{~mW}$ with $1.2 \mathrm{~V}$ supply and clock frequency of $3 \mathrm{GHz}$.
\end{abstract}

Keywords- capacitive DAC, high speed DAC, highly linear output driver.

\section{INTRODUCTION}

On the pace towards broadband connectivity in wireless telecommunication systems increasingly more demands are placed on the performance and speed of the data converters. To enable ever higher data rates, wider channel bandwidths and advanced DSP techniques such as OFDM or multi-bit QAM are required that in terms of design, transform onto speed, dynamic range, and the linearity specifications. Implemented in CMOS technology data converters have proven to meet the broadband communication challenges in particular for the speed of CMOS devices and also for low manufacturing cost .

Among D/A converters (DACs), recently, the currentsteering architectures [1-4] have been mainly focused on due to their simplicity and the achieved good performances. Unlike this, the capacitive DACs (SC DACs) have not drawn much attention except for two recent publications $[5,6]$.

In fact, SC DACs have some advantages over the currentsteering architectures [5, 6]. Specifically, as capacitor arrays they are easier to match that makes SC DACs virtually more linear. For $n$-bit resolution a pipeline SC DAC only requires $n$ identical stages as opposed to $2^{n}$ unit cells in a current steering DAC. Also the power consumption is low because their operation is based on charge redistribution. On the other hand, the ability to drive off-chip load of SC DACs is poor, so a suitable output driver is necessary. The driver appears critical for linearity of a capacitive DAC unless the required output voltage swing is low. One solution to this problem is using a closed-loop architecture where the circuit linearity is improved by negative feedback [5]. This technique, however, cannot work well at high frequencies $(>1 \mathrm{GHz})$. Another solution is an open-loop driver design that can be supported by interleaving and a pre-distortion technique [6]. However, the latter is usually limited by an assumption of minimum memory effect of the nonlinear object.

In this paper we present design of a simple pipeline $\mathrm{SC}$ DAC with a highly linear output driver using $65 \mathrm{~nm}$ CMOS technology. The main objective is achieving maximum data rate in this architecture. The circuit is optimized under linearity and noise constraints imposed by the capacitor array and the output driver. We define the design area of the SC array in terms of unit capacitance and the switch size for given resolution and clock frequency. The SFDR and SNR analysis shows that the DAC bandwidth limit is subject to incomplete settling and feed-through effects rather than $\mathrm{kT} / \mathrm{C}$ noise and the capacitor mismatch. A tradeoff between the DAC bandwidth and resolution accompanied by SFDR is demonstrated.

The DAC output driver is an open-loop wideband amplifier patterned after [22]. To attain high linearity a combination of the derivative superposition [12, 14] and resistive source degeneration technique is used. We estimate the driver nonlinearity by using the Volterra series model [23]. The analysis verified by simulation shows that for signal bandwidth up to $5 \mathrm{GHz}$ and the output voltage swing $300 \mathrm{mV}_{\mathrm{pp}}$, HD3 better than $-72 \mathrm{~dB}$ is attained that corresponds to $12 \mathrm{bit}$ resolution. This shows the contribution of the SC array to HD3 and SFDR of the SC DAC to be dominant.

Simulation results of the complete SC DAC with 8-bit resolution and Nyquist sampling show SFDR of $45 \mathrm{~dB}$ for 1.36 $\mathrm{GHz}$ signal bandwidth. For 6-bit resolution and signal bandwidth of $5.5 \mathrm{GHz}$, SFDR of $33 \mathrm{~dB}$ is attained. The respective maximum data rate of 72 Gbps compares favorably with the state-of-the-art.

The paper is arranged as follows. In Section II the pipeline SC DAC architecture and its operation principle are presented. Analysis of the SC array in terms of noise, capacitor mismatch, clock feed-through, and SC settling time is provided in Section III. Based on this we introduce the DAC design area model to identify the maximum data rates and SFDR feasible in $65 \mathrm{~nm}$ CMOS technology. Comparisons to the possible $28 \mathrm{~nm}$ CMOS implementation are provided in Appendix. In Section IV we present the output driver design supported by Volterra model linearity analysis and noise analysis. Section $\mathrm{V}$ provides simulation results of the complete SC DAC, followed by a discussion. Conclusions are formulated in the last section. 


\section{SC DAC ARCHITECTURE}

The pipeline capacitive DAC architecture shown in Fig.1 was firstly proposed in [18]. The 12-bit input is divided into 4 segments of three-bits each. It needs one cycle of clock to complete 3-bit conversion in the 3-bit SC circuits. The 3-bit DFFs are employed to synchronize the signal using a nonoverlapping three-phase clock. The output driver serves as a buffer driving an off-chip standard load.

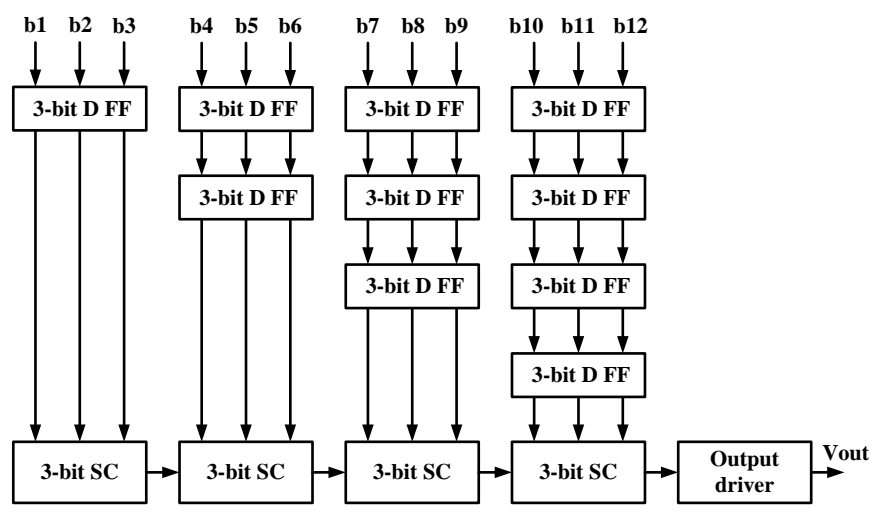

Figure 1. Pipeline SC DAC architecture.

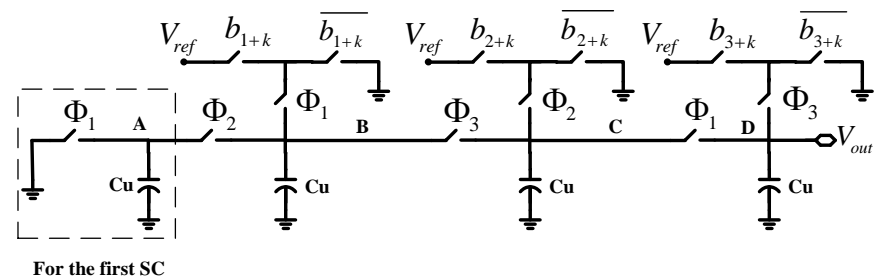

Figure 2. Three-bit switched capacitor segment.

Figure 2 depicts the 3-bit SC segment where a three-phase clock $\left(\Phi_{1}, \Phi_{2}\right.$ and $\left.\Phi_{3}\right)$ is used. In the first SC segment, an extra switch is necessary to reset voltage at node $\mathrm{A}$ in the pre-charge step. During the time when $\Phi_{1}=1$, the capacitor at $\mathrm{A}$ is discharged to zero and the capacitor at $\mathrm{B}$ is charged either to $V_{\text {ref }}$ if $b_{1+k}=1$ or discharged if $b_{1+k}=0$. When $\Phi_{1}$ goes low and $\Phi_{2}$ goes high, the voltages at $\mathrm{A}$ and $\mathrm{B}$ are equal to $V_{r e f} b_{1+k} / 2$. The capacitor at node $\mathrm{C}$ is controlled like the cap at $\mathrm{B}$ in phase $\Phi_{1}$. Next, when the clock $\Phi_{3}=1$ the voltage at B and C would attain a level of $V_{\text {ref }}\left(b_{1+k} / 2+b_{2+k}\right) / 2$. Similarly, when $\Phi_{1}=1$ again, the voltages at $\mathrm{C}$ and $\mathrm{D}$ will be equal to $V_{r e f}\left(b_{1+k} / 4+b_{2+k}\right.$ $\left./ 2+b_{3+k}\right) / 2$. This is in fact, the output voltage of the first SC segment and the same process will continue for the following segments.

When all the parasitic capacitances of the circuit are neglected the output voltage of the SC pipeline DAC is linearly dependent on the values of input data according to

$$
V_{\text {out }}=V_{\text {ref }} \sum_{i=0}^{n-1} \frac{b_{i}}{2^{n}} 2^{i}
$$

\section{SC ARRAY DESIGN AND ANALYSIS}

\section{Noise analysis}

Noise contribution from all switches to the output can be estimated using the superposition principle. Basically the hold portion of noise is considered since the track noise is not transferred except for two switches in the last section of the pipeline. For switch $\Phi_{1}$ of the first section (Fig. 3) the voltage transfer function to the output is $1 / 2^{n}$, where $n$ is the number of capacitors (DAC bits) in the pipeline. Its noise power spectral density (PSD) is subject to folding with a factor $1 /\left(2 R_{o n} C_{u} f_{s}\right)$ [20] due to the equivalent noise bandwidth $1 /\left(4 R_{o n} C_{u}\right)$ and switching frequency $f_{s}$, where $R_{o n}$ denotes the switch on-resistance.

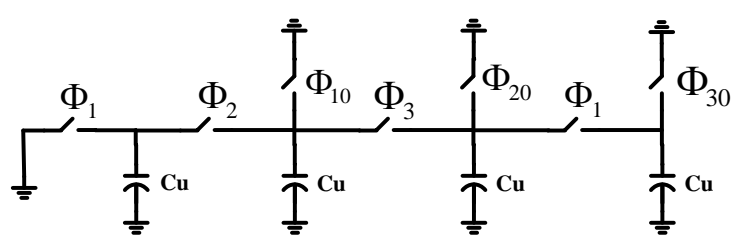

Figure 3. Model of first section of SC pipeline.

Moreover, we observe that the transferred noise voltage remains on the output capacitor only for $2 / 3$ part of the clock period and otherwise the capacitor is discharged. The latter effect is reflected in the frequency domain by a sinc function shaping the noise spectrum accordingly. Hence, the respective noise PSD contribution (one sided) to the output at low frequency can be found from

$$
S_{1}(f)=4 k T R_{o n} \times \frac{1}{2 R_{o n} C_{u} f_{s}} \times \frac{1}{2^{2 n}} \times\left(\frac{2}{3}\right)^{2}
$$

The contribution of switch $\Phi_{10}$ is the same as of $\Phi_{1}$. For noise of the $\Phi_{2}$ switch we find the incident capacitance to be $C_{u} / 2$ and the respective held voltage to be shared by two $C_{u}$ capacitors in series. The transfer function to the output is $1 / 2^{n-1}$ so the noise contribution of $\Phi_{2}$ switch appears as

$$
S_{2}(f)=4 k T R_{o n} \times \frac{1}{R_{o n} C_{u} f_{s}} \times \frac{1}{2^{2}} \times \frac{1}{2^{2(n-1)}} \times\left(\frac{2}{3}\right)^{2}
$$

For switch $\Phi_{3}$ only the transfer function is changed to $1 / 2^{n-2}$ so $S_{3}(f)=S_{2}(f) / 4$.

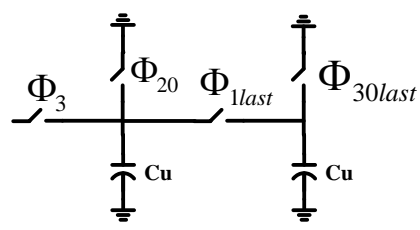

Figure 4. Model of last section of SC pipeline.

For the last two switches in the pipeline (Fig.4) also their track noise should be taken into account 


$$
\begin{aligned}
& S_{\text {last }}(f)=4 k T R_{o n} \times \frac{1}{R_{s w} C_{u} f_{s}} \times \frac{1}{2^{2}} \times\left(\frac{1}{3}\right)^{2}+4 k T R_{o n} \times \frac{1}{2^{2}} \times \frac{1}{3} \\
& S_{3 l a s t}(f)=8 k T R_{o n} \times \frac{1}{4 R_{s w} C_{u} f_{s}} \times \frac{1}{2^{2}} \times\left(\frac{2}{3}\right)^{2}+8 k T R_{o n} \times \frac{1}{3}
\end{aligned}
$$

where for $\Phi_{\text {1last }}$ contribution both the hold and track time is $1 /\left(3 f_{s}\right)$. For $\Phi_{3 \text { last }}$ a resistance of $2 R_{o n}$ is assumed (two switches in series). The total hold noise by the floating switches $\Phi_{2}, \Phi$ $3, \Phi_{1}, \ldots$ can be estimated as

$$
S_{f}(f)=\frac{4 k T}{9 C_{u} f_{s}} \times\left(\sum_{k=1}^{n-1} \frac{1}{2^{2 k}}+\frac{1}{4}\right) \cong \frac{7 k T}{27 C_{u} f_{s}}
$$

and the hold noise contribution by the remaining switches (to ground or reference source) is

$$
S_{g}(f)=\frac{8 k T}{9 C_{u} f_{s}} \times\left(\sum_{k=1}^{n} \frac{1}{2^{2 k}}+\frac{1}{4}+\frac{1}{2^{2 n}}\right) \cong \frac{14 k T}{27 C_{u} f_{s}}
$$

The total noise PSD at the output including the track noise can be found from

$$
S_{\text {tot }}(f) \cong \frac{7 k T}{9 C_{u} f_{s}}+3 k T R_{o n}
$$

For practical design values the hold noise (kT/C) in (7) matters while the track noise term in can be omitted.

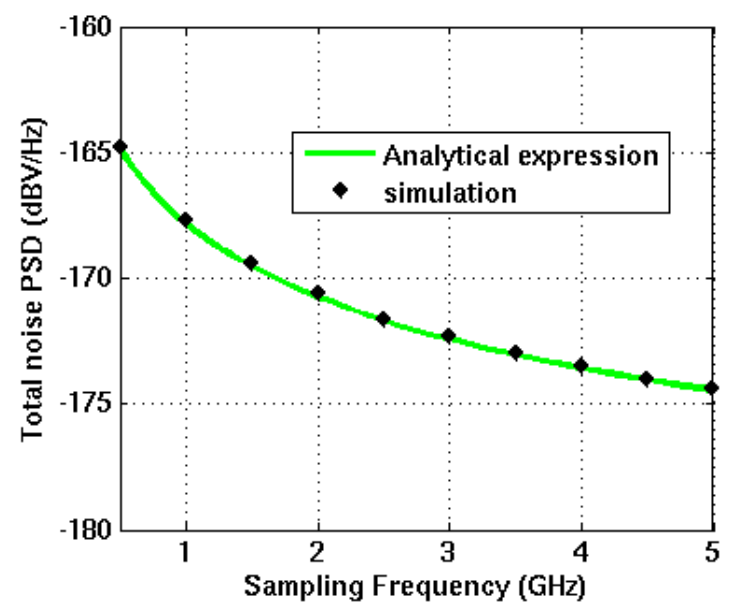

Figure 5. SC noise PSD comparison of analytical model (7) and SpectreRF ${ }^{\mathrm{TM}}$ simulations with $C_{u}=200 \mathrm{fF}, R_{o n}=30 \Omega$.

Figure 5 shows a comparison of SC noise PSD obtained with (7) and by a circuit simulation model using SpectreRFTM. The results are achieved by Transient noise simulation followed by spectrum averaging at low frequencies. Using Pnoise analysis the same result is obtained.

Generally, the SC noise should be less than quantization noise of DAC which can be calculated from

$$
S_{Q}(f)=\frac{V_{F S}^{2}}{12\left(2^{n}-1\right)^{2} B W}
$$

where $V_{F S}$ is the full-scale voltage, $n$ is the number of bits, and $B W=f_{s} / 2$. Then using (7) we can write

$$
\frac{7}{9} \frac{k T}{C_{u} f_{s}}<\frac{V_{F S}^{2}}{12 B W\left(2^{n}-1\right)^{2}}
$$

that can be seen as a design constraint for the unit capacitance $C_{u}$

$$
C_{u}>\frac{4.67 k T\left(2^{n}-1\right)^{2}}{V_{F S}^{2}}
$$

For example, for $n=12$ and $V_{F S}=1.3 \mathrm{~V}$, and $T=290 \mathrm{~K}$ from (10) we obtain $C_{u}>191 \mathrm{fF}$. Observe, that for the minimum value of $C_{u}$ the total noise level of DAC gets doubled and SNR is less by $3 \mathrm{~dB}$ compared to SNQR.

\section{Capacitor mismatch analysis:}

Following the analysis in [21] a similar estimate for voltage error due to capacitor mismatch can be attained

$$
V_{D N L} \approx \frac{\sqrt{2^{n}-1}}{2^{n}} \frac{\Delta C_{u}}{C_{u}} V_{F S}
$$

where $V_{F S}$ is the full-scale output range and $V_{D N L}$ is the differential nonlinearity of DAC. The standard deviation $\sigma\left(\Delta C_{u} / C_{u}\right)$ of the capacitor mismatch for a typical metalinsulator-metal (MIM) capacitor can be found from [24]

$$
\sigma\left(\frac{\Delta C_{u}}{C_{u}}\right)=\frac{K_{\sigma}}{\sqrt{A}} \quad \text { and } \quad C_{u}=K_{C} A
$$

where $A$ is the capacitor areawhereas $K_{\sigma}$ (matching coefficient) and $K_{C}$ (capacitance density) are technology constants provided by the chip manufacturer. From (11) and (12), the standard deviation of the voltage error caused by capacitor mismatch is found as

$$
\sigma\left(V_{D N L}\right) \approx \frac{\sqrt{2^{n}-1}}{2^{n}} V_{F S} \sigma\left(\frac{\Delta C_{u}}{C_{u}}\right)
$$

For the DAC accuracy, it is common to maintain $3 \sigma\left(V_{D N L}\right)$ less than a half LSB. Hence, the lower bound for the unit capacitor due to the mismatch in $n$-bit DAC is

$$
C_{u}>9 K_{C} K_{\sigma}^{2}\left(2^{n}-1\right)
$$

For $65 \mathrm{~nm}$ CMOS process we have $K_{\sigma}=0.5 \% \mu \mathrm{m}, K_{C}=5$ $\mathrm{fF} / \mu \mathrm{m}^{2}$ that for 12-bit DAC give the unit capacitor $C_{u}>5 \mathrm{fF}$. As already shown, the limit due to $\mathrm{kT} / \mathrm{C}$ noise calculated from (10) for $V_{F S}=1.3 \mathrm{~V}$ is $C_{u}>191 \mathrm{fF}$ so it largely prevails while the limit due to the capacitor mismatch can be neglected.

To verify the estimates by simulation, we refer to more accurate equations from [21]

$$
V_{D N L}=\frac{C_{u}+\left(\sqrt{2^{n-1}}-\sqrt{2^{n-1}-1}\right) \Delta C_{u}}{2^{n} C_{u}+\sqrt{2^{n}} \Delta C_{u}} V_{F S}-\frac{V_{F S}}{2^{n}}
$$

The standard variation of $V_{D N L}$ would be 


$$
\sigma\left(V_{D N L}\right)=\frac{1+\left(\sqrt{2^{n-1}}-\sqrt{2^{n-1}-1}\right) \sigma\left(\frac{\Delta C_{u}}{C_{u}}\right)}{2^{n}+\sqrt{2^{n}} \sigma\left(\frac{\Delta C_{u}}{C_{u}}\right)} V_{F S}-\frac{V_{F S}}{2^{n}}
$$

From (12), we have

$$
\sigma\left(\frac{\Delta C_{u}}{C_{u}}\right)=\frac{K_{\sigma} \sqrt{K_{C}}}{\sqrt{C_{u}}}
$$

Substituting (17) to (16), we have

$$
\sigma\left(V_{D N L}\right)=\frac{1+\left(\sqrt{2^{n-1}}-\sqrt{2^{n-1}-1}\right) \frac{K_{\sigma} \sqrt{K_{C}}}{\sqrt{C_{u}}}}{2^{n}+\sqrt{2^{n}} \frac{K_{\sigma} \sqrt{K_{C}}}{\sqrt{C_{u}}}} V_{F S}-\frac{V_{F S}}{2^{n}}
$$

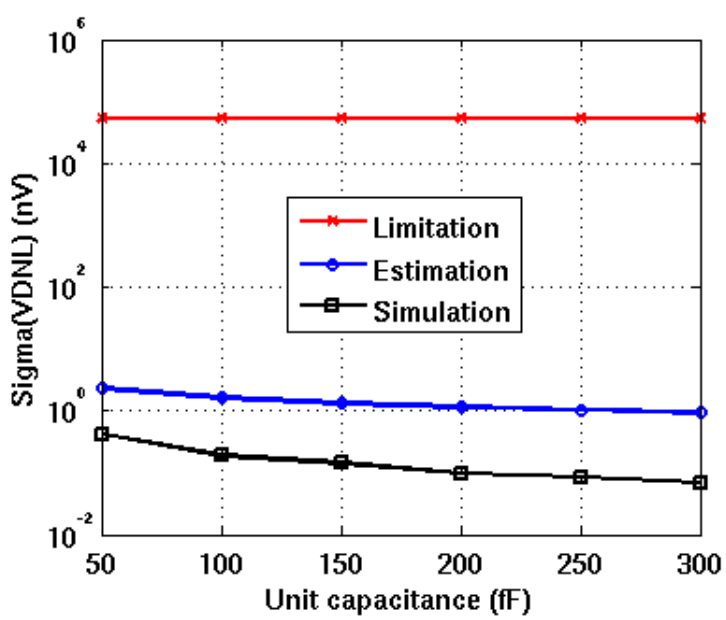

Figure 6. $\quad \mathrm{V}_{\mathrm{DNL}}$ standard deviation comparison of estimated model (18) and SpectreRF ${ }^{\mathrm{TM}} \mathrm{MC}$ simulations against $1 / 6 \mathrm{LSB}$ voltage of 12 bit DAC.

With $65 \mathrm{~nm}$ CMOS process of STM foundry we have $K_{\sigma}=$ $0.5 \% \mu \mathrm{m}, K_{C}=5 \mathrm{fF} / \mu \mathrm{m}^{2}$. Fig. 6 gives a comparison of $\mathrm{V}_{\mathrm{DNL}}$ standard deviation obtained with model (18) and SpectreRFTM simulations. The simulation results are achieved by statistical Monte-Carlo analysis only with the focus on capacitor variation whereas the switches are assumed ideal and the simulation count is 100 . A very low clock frequency was used to verify the static $\mathrm{V}_{\mathrm{DNL}}$ with input data of MSB transition [21]. We usually use $3 \sigma$ standard, meaning that $3 \sigma\left(V_{D N L}\right)$ should be less than a half of $V_{\text {LSB }}$. The limitation line shown in Fig. 6 is therefore one sixth of $V_{\text {LSB }}$ of 12-bit resolution DAC which is $53 \mu \mathrm{V}$ with $V_{F S}=1.3 \mathrm{~V}$. The $\mathrm{V}_{\mathrm{DNL}}$ standard deviations are in nano-volt range while those from simulation are by one order of magnitude less than this. The results from simulation are lower than the ones from estimation because in calculation we assumed total variation of mismatch as maximum [21] while the variation of unit capacitors mismatch in Monte-Carlo simulation is randomly distributed. Importantly, the both values are by orders of magnitude less than the limitation voltage of 12-bit DAC. Therefore the limit due to the capacitor mismatch can be neglected while the one due to $\mathrm{kT} / \mathrm{C}$ noise prevails in this case.

\section{Clock feed-through effect:}

To discuss the feed-through effect we consider three groups of switches in the SC array (Fig. 2), namely: data switches, charge/discharge switches, and charge redistribution switches. The clock feed-through caused by the data switches is not meaningful because there is always a low time-constant path either to $V_{\text {ref }}$ or $G N D$. Unlike this, the feed-through related to the charge switches changes the capacitor voltage when they are switched off because the respective capacitors see high impedance during this period of time. Since the switches are designed as transmission gates, there is inherent partial cancelation of this effect due to the rising and falling edge applied at the same time to the PMOS and NMOS device, respectively. This cancelation requires correction by dummy transistors [19] since the PMOS devices are three times larger than the NMOS (for balance of resistance).

The charge redistribution switches require compensation of the feed-through effect not only upon switching off but also switching on, taking thereby more effect on the transfer function and the DAC linearity. For this reason the redistribution switches require more precise feed-through cancelation that also entails an extra design constraint. Assuming the feed-through voltage to be limited by a half LSB we have

$$
K_{d i f} K_{\text {comp }} \frac{C_{P}(W)}{C_{u}+C_{P}(W)} V_{d d}<\frac{V_{r e f}}{2^{n+1}}
$$

where $C_{p}(W)$ is parasitic capacitance of a switch with respect to the switch size, while $K_{\text {comp }}$ is a constant representing the feedthrough compensation by the dummy devices. This constant can be identified during optimization of the charge redistribution switches. Assuming that the total mismatch between two single-ended branches of differential topology (including mismatch of switches, caps, jitter, skew) is $K_{d i}$, from (19) the limit for $C_{u}$ follows

$$
C_{u}>\left(K_{d i f} K_{\text {comp }} 2^{n+1} \frac{V_{d d}}{V_{\text {ref }}}-1\right) C_{P}(W)
$$

\section{Settling time analysis}

Due to imperfections of the transistor switches $\left(R_{\text {off }} / R_{\text {on }}\right.$ resistances) the leakage and incomplete settling in the capacitor array result in voltage errors which degrade DAC linearity [18]. Using the schematic in Fig. 2, we can consider an error at node B for $\Phi_{1}=0$ while $\Phi_{2}=1$. For the incident bit value equal to 1 , there is a leakage current from $V_{\text {ref }}$ through $R_{\text {off }}$ to the capacitance $2 C_{u}$ seen from node B. In this case, the onresistance of switch $\Phi_{2}$ can be neglected since $R_{\text {on }}<<R_{\text {off. }}$. The respective voltage change (Fig. 7a) can be expressed as

$$
V_{C 1}=V_{\text {ref }}\left(1-e^{\frac{-t_{\text {puls }}}{2 R_{\text {of }}}}\right)
$$

where $R_{\text {off }}$ is the off-resistance of a charge switch, $T_{s}=1 / f_{s}$ is clock period, and $t_{\text {pulse }}$ is the pulse width. Importantly, 
$t_{\text {pulse }}<1 /\left(3 f_{s}\right)$ to guarantee non-overlapping 3-phase clock operation. In order to keep the voltage error (21) less than half LSB we find the lower bound of $R_{\text {off }}$

$$
R_{\text {off }}>-\frac{t_{\text {pulse }}}{2 C_{u}} \frac{1}{\ln \left(1-2^{-n-1}\right)}
$$

Similarly, we can find the upper bound of the switch onresistance. Fig. 7 (b) shows the charge phase with incident input bit equal to 1 . The respective settling time error can be found from

$$
V_{C 2}=V_{r e f} e^{\frac{-t_{\text {puse }}}{2 R_{o n} C_{u}}}
$$

This error should also be limited by half LSB that leads to

$$
R_{\text {on }}<\frac{t_{\text {pulse }}}{2 C_{u}} \frac{1}{\ln \left(2^{n+1}\right)}
$$

For completeness, we should also consider an error during charge redistribution phase shown in Fig. 7 (c). To calculate $V_{B}$, we use superposition

$$
V_{B}=\frac{V_{A 0}}{2}\left(1-e^{\frac{-t_{\text {pulse }}}{T}}\right)-\frac{V_{B 0}}{2}\left(1-e^{\frac{-t_{\text {pulse }}}{T}}\right)+V_{B 0}
$$

where $V_{A 0}$ and $V_{B 0}$ are initial values of $V_{A}$ and $V_{B}$, respectively. The time constant $T=R_{o n} C_{u} / 2$. In the worst case, for $V_{A 0}=0$ and $V_{B 0}=V_{\text {ref }}$ this voltage will be

$$
V_{B}=\frac{V_{r e f}}{2}\left(1+e^{\frac{-2 t_{p u l s e}}{R_{o n} C_{u}}}\right)
$$

As the error voltage $\left(V_{B^{-}} V_{r e f} / 2\right)$ should be less than half LSB

$$
R_{o n}<\frac{1}{\ln \left(2^{n-1}\right)} \frac{2 t_{\text {pulse }}}{C_{u}}
$$

Since (27) is more relaxed compared to (24) the upper bound for $R_{o n}$ is set by (24).

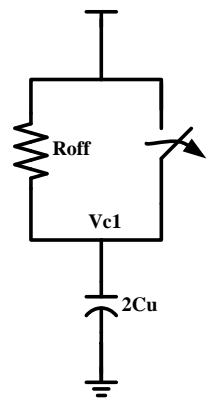

(a)

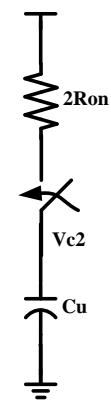

(b)

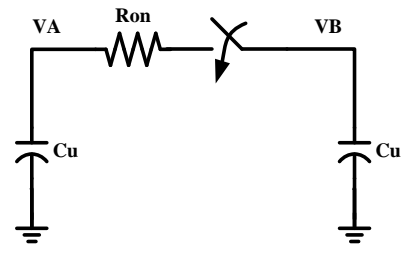

(c)
Figure 7. Charged and discharged circuits: (a) Leakage due to off-resistance of data switches, (b) Incomplete charge due to on-resistance of data switches (c) Incomplete charge redistribution due to inbetween switches.

The settling conditions (22) and (24) appear stringent for high frequency clock. For illustration, let us consider $f_{s}=3$
$\mathrm{GHz}, t_{\text {pulse }}=50 \mathrm{ps},\left(t_{\text {pulse }}<1 /\left(3 f_{s}\right)\right), n=7$, and $C_{u}=200 \mathrm{fF}$. In this case the lower bound of $R_{\text {off }}=30 \mathrm{k} \Omega$ and the higher bound of $R_{o n}=19.5 \Omega$. A suitable size of a switch (transmission gate) in $65 \mathrm{~nm}$ CMOS with channel length $L=0.06 \mu \mathrm{m}$ is depicted in Fig. 8. The width range of $\left[W_{\min }, W_{\max }\right]=[150,2000] \mu \mathrm{m}$ satisfies the the design constraints (22) and (24). However, for higher DAC resolutions the available design range is reduced and already for $n>8$, it will vanish unless the demand of half LSB accuracy is relaxed.

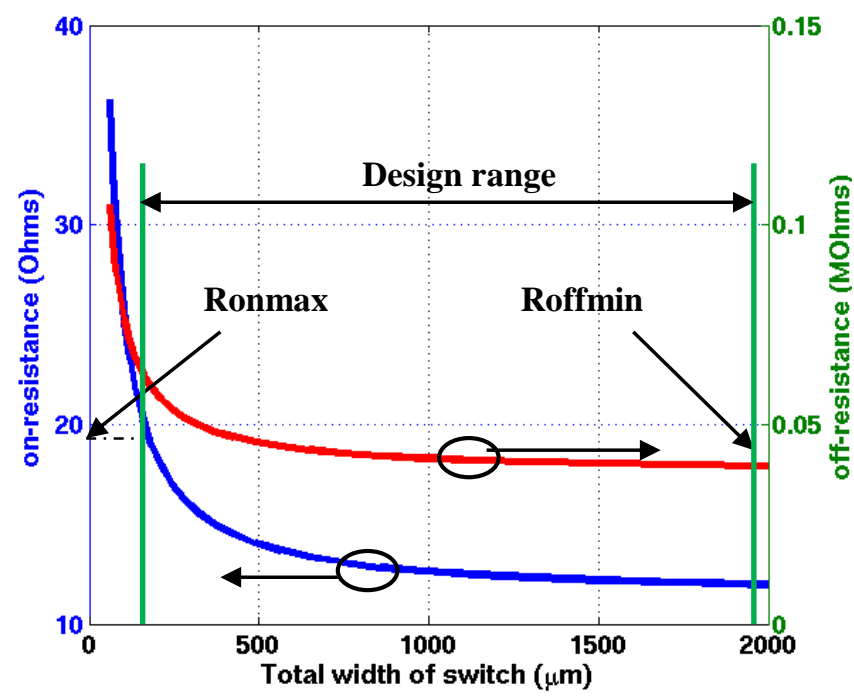

Figure 8. Off-resistance and on-resistance of T-gate switch vs. width in $65 \mathrm{~nm}$ CMOS with $L=0.06 \mu \mathrm{m}\left(W_{p} / W_{n}=3\right)$.

\section{Bounds for unit capacitor:}

Having mapped the resistances $R_{\text {off }}$ and $R_{o n}$ onto the width of the switching devices, we can identify functions $R_{\text {off }}(W)$ and $R_{o n}(W)$, and redefine our design task by combing (22) and (24) as follows

$$
-\frac{t_{\text {pulse }}}{2 R_{\text {off }}(W)} \frac{1}{\ln \left(1-2^{-n-1}\right)}<C_{u}<\frac{t_{\text {pulse }}}{2 R_{\text {on }}(W)} \frac{1}{\ln \left(2^{n+1}\right)}
$$

In this case, the lower- and upper bound for $C_{u}$ are defined in terms of the switch size $W$, pulse time $t_{\text {pulse }}$, and the number of bits $n$. In fact, also $t_{\text {pulse }}$ depends on $W$ due to parasitic capacitance of a switch and the driving source resistance that reduce the pulse time. Estimating the rise/fall time $t_{r f}$ from $10 \%$ to $90 \%$ voltage change we find

$$
t_{r f}=\ln (9) R_{d} C_{g}(W)
$$

where $R_{d}$ is the driver resistance and $C_{g}(W)$ is the gate capacitance with respect to the switch width $W$. Then $t_{\text {pulse }}$ time appears as

$$
t_{\text {pulse }}=\frac{1}{3 f_{S}}-t_{n}-4 \ln (9) R_{d} C_{g}(W)
$$

where $t_{n}$ is a latency time to guarantee non-overlapping clock. The additional reduction by $4 \times t_{r f}$ is due to the rising and falling time, while $C_{g}$ value is doubled due to a dummy switch (to compensate for the feed-through effect [19]). Based on this (28) can be rewritten as 


$$
\frac{\left(3 f_{S}\right)^{-1}-t_{n}-8.8 R_{d} C_{g}(W)}{-2 \ln \left(1-2^{-n-1}\right) R_{o f f}(W)}<C_{u}<\frac{\left(3 f_{S}\right)^{-1}-t_{n}-8.8 R_{d} C_{g}(W)}{2 \ln \left(2^{n+1}\right) R_{o n}(W)}
$$

This result is illustrated in Fig. 9 for clock frequency $f_{s}=$ $3 \mathrm{GHz}, t_{n}=30 \mathrm{ps}, n=7$ with solid lines representing the upper and lower bounds for $C_{u}$. The design area $W-C_{u}$ is additionally limited by the feed-through condition (20) shown as the straight line (green) lower bound. The conditions due to $\mathrm{kT} / \mathrm{C}$ noise and capacitor mismatch are negligible in this case. For $W$ $<570 \mu \mathrm{m}$ the $C_{u}$ lower bound due to $R_{\text {off }}$ applies. For $W>$ $570 \mu \mathrm{m}$ and $W<780 \mu \mathrm{m}$ the lower bound due to the feedthrough limit applies.

The dashed lines illustrate the design area for the 8-bit resolution to vanish (the upper bound goes below the lower bound). If the accuracy requirement of half LSB is relaxed the design area can be restored. Interestingly, for 1-LSB accuracy and $n$-bit resolution the same design area is achieved as for $(n-1)$-bit with half-LSB accuracy, so the design area shown in Fig. 9 for the 7-bit DAC is also valid for the 8-bit provided the accuracy constraints are changed to 1-LSB.

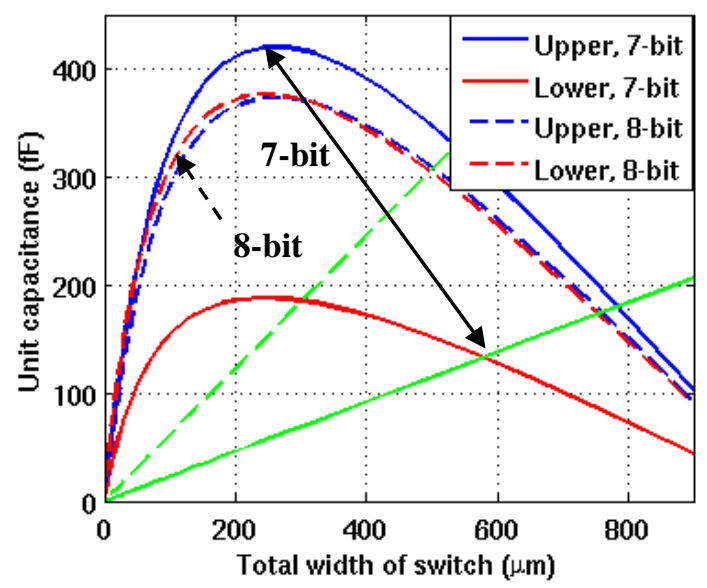

Figure 9. Design area $\mathrm{W}-\mathrm{C}_{\mathrm{u}}$ for 7-bit and 8-bit SC DAC, $f_{S}=3 \mathrm{GHz}, K_{\text {dif }}=20 \%$ and $K_{\text {comp }}=1 \%$.

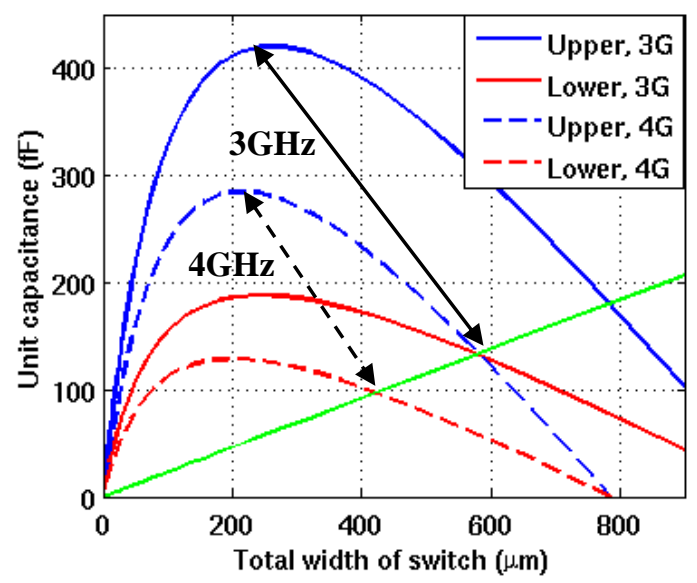

Figure 10. Design area $\mathrm{W}-\mathrm{C}_{\mathrm{u}}$ for 7-bit SC DAC with different clock frequency $\left(f_{s}\right)\left(K_{\text {dif }}=20 \%, K_{\text {comp }}=1 \%\right)$.

According to (31) the design area largely shrinks with the clock frequency as well. This is illustrated in Fig. 10 when the clock frequency is increased from $3 \mathrm{GHz}$ to $4 \mathrm{GHz}$ for $n=7$. In this case the feed-through bound is not changed but the other two bounds due to (31), go down largely reducing the design area.

To increase the speed of this SC DAC, we have to reduce its resolution. For example, increasing the clock frequency to $f_{s}$ $=12 \mathrm{GHz}$ a convenient design area for a 6-bit DAC is available as shown in Fig. 11. Then, with the corresponding Nyquist bandwidth $<6 \mathrm{GHz}$ the estimated maximum data rate amounts for $72 \mathrm{Gbps}$.

In the analysis and simulations, the reference voltage was assumed as an ideal source. However, previous work such as [4] has shown that the reference voltage can for example be generated using on-chip low drop-out regulator (LDO).

In principle, the tradeoff between the resolution and bandwidth (or data rate) in this design can be conveniently moved towards higher levels of those quantities by making use of newer CMOS technologies. For illustration in Appendix-C we demonstrate the DAC design areas for Fully Depleted Silicon-on-Insulator (FD SOI) $28 \mathrm{~nm}$ CMOS process that enables a significant increase of the resolution and bandwidth. Clearly, the tradeoff between them still exists. However, other features such as better element matching, compared to current steering solutions, make SC DACs even more appealing.

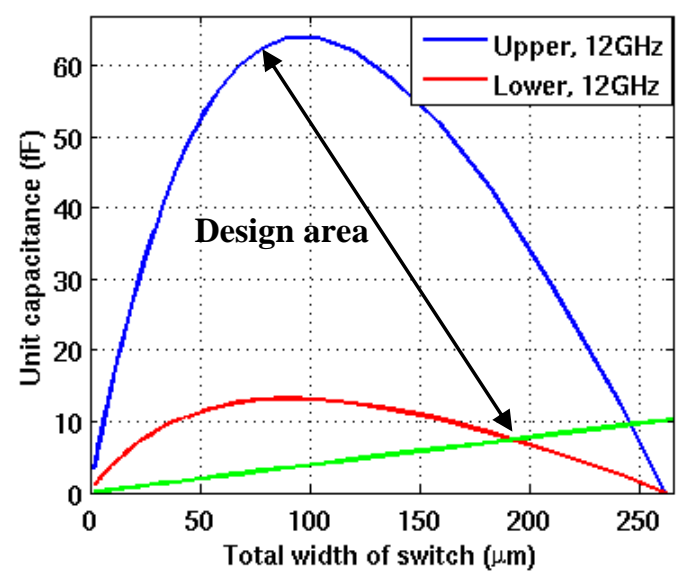

Figure 11. Design area $\mathrm{W}_{-} \mathrm{C}_{\mathrm{u}}$ for 6-bit SC DAC at $12 \mathrm{GHz}$ clock frequency.

\section{OUTPUT DRIVER DESIGN}

\section{Problem and solution:}

The output voltage of high speed DACs for wireless and wire LAN communications can also exceed $300 \mathrm{mV}_{\mathrm{pp}}$. In practice, we have to treat this voltage as a large signal like in the power amplifier case. There are several linearization techniques well established in power amplifier design [8]. Predistortion as one of them was already applied in a high speed DAC to compensate for non-linearity of its output driver [6]. Using the main and auxiliary amplifier/driver for non-linearity cancellation [9, 10] is another possible solution. As an additional inspiration the techniques developed for low noise amplifiers (LNAs) can also be considered [11-14, 17]. In particular, the third-order distortions can be cancelled using various linearization techniques, like negative feedback, 
harmonic termination, optimum biasing, feed-forward, or derivative superposition (DS).

In this design, we are targeting high SFDR and bandwidth (> $1 \mathrm{GHz})$. This makes the driver design a challenge. Fig. 12 (a) shows a circuit suitable for third-order non-linearity cancellation by DS method that has been recognized both in small- and large signal applications such as LNAs [12] and power amplifiers [16], respectively. This technique is based on cancellation of the transconductance $g_{3}\left(\mathrm{~d}^{3} I_{d} / \mathrm{d}\left(V_{g s}\right)^{3}\right)$ of the main transistor and $g_{3}$ of the auxiliary one for properly biased devices.

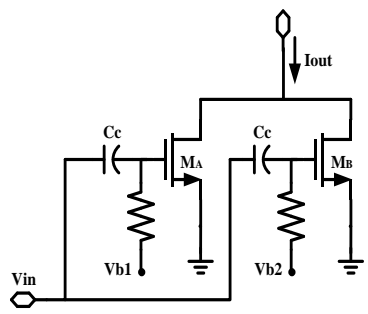

(a)

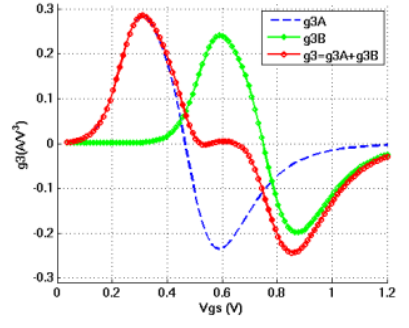

(b)
Figure 12. (a) DS technique with dual-NMOS. (b) Third-order distorion terms of the main transistor $\left(\mathrm{g}_{3 \mathrm{~A}}\right)$, auxiliary transistor $\left(\mathrm{g}_{3 \mathrm{~B}}\right)$, and total output $\left(\mathrm{g}_{3}\right)$ using ST 65nm CMOS process, $(W / L)_{\mathrm{MA}}=20 / 0.065 \mu \mathrm{m}$, $(W / L)_{\mathrm{MB}}=17 / 0.065 \mu \mathrm{m}, V_{d s}=1.2 \mathrm{~V}$ and $V_{\text {shift }}=282 \mathrm{mV}$.

As shown in Fig. 12 (b), if $V_{g s}$ is biased around $[0.52-0.67] \mathrm{V}$ the output current has negligible $3^{\text {rd }}$ order transconductance. So the suitable input swing is about 150 $\mathrm{mV}_{\mathrm{pp}}$ that is however, not sufficient for most applications. Moreover, the auxiliary transistor has to be biased in weak inversion to generate positive $g_{3 \mathrm{~B}}$ value [14]. Larger input swing tend to push this transistor into the off region for a part of the input cycle raising the distortion. With $300 \mathrm{mV}_{\mathrm{pp}}$ swing this circuit cannot provide HD3 better than $-50 \mathrm{~dB}$.

To overcome these problems, we propose a combination of a simple inherent feedback and DS technique that is illustrated in Fig. 13. The negative feedback technique helps to reduce both second- and third order non-linearity of a system [14]. Specifically, we try to avoid using inductors and complexity by complementary circuits necessary in different solutions.

Like for the inductive source-degeneration [14], in this case $A_{I I P 3}$ improves approximately by a ratio $\left(1+g_{m 1} R_{S}\right)^{3 / 2}$. Moreover, the source resistor provides wideband matching for the driver load.

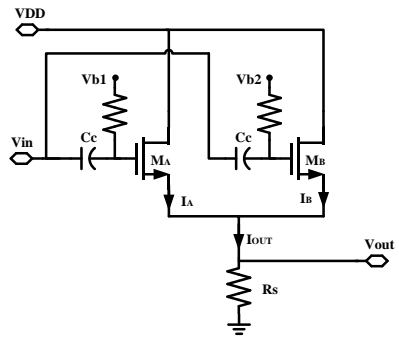

(a)

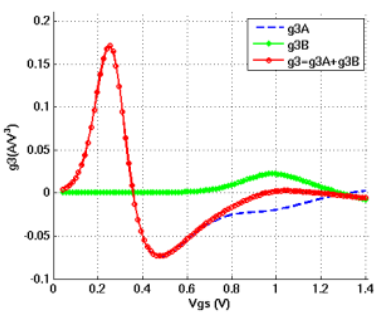

(b)
Figure 13. (a) DS technique with resistive feedback. (b) Third-order distorion terms of the main transistor $(\mathrm{g} 3 \mathrm{~A})$, auxiliary transistor $\left(\mathrm{g}_{3 \mathrm{~B}}\right)$, and total output $\left(\mathrm{g}_{3}\right)$ using ST 65nm CMOS process, $(W / L)_{\mathrm{MA}}=20 / 0.065 \mu \mathrm{m},(W / L)_{\mathrm{MB}}=$ $17 / 0.065 \mu \mathrm{m}, V_{d s}=1.4 \mathrm{~V}$ and $V_{\text {shift }}=452 \mathrm{mV}$.
A large value of $g_{m 1} R_{S}$ is also useful to maximize the closed-loop voltage gain of the driver, ideally approaching one. Since the effective $R_{S}$ value is limited by the $50 \Omega$ load, more gain can only be achieved by increasing $g_{m 1}$ at the expense of the power consumption.

Fig. 13 (a) shows a half of the proposed output driver designed as a differential circuit. The voltage at the output of $\mathrm{SC}$ pipeline can be large enough to provide around $300 \mathrm{mV}_{\mathrm{pp}}$ at the driver output even for a moderate gain $g_{m 1} R_{S}$. Since we use $1.2 \mathrm{~V}$ supply to limit power consumption of the driver, not much voltage headroom is left to keep the signal within a linear region of the input/output characteristics. Inevitably, the signal is exposed to non-linear behavior of the transistors and hence, good enough cancelling of the non-linear effects is necessary.

As shown in Fig. 13 (b) the cancelling range is much wider compared to the previous case. The both transistors operate in saturation that makes the circuit less sensitive to large input swing. Also larger bias voltages are allowed providing larger transconductance values $g_{m 1}$ and lower HD3 distortions at the same time.

\section{Volterra series model:}

The advantage of the DS technique combined with the resistive feedback will be discussed using the Volterra series model [23]. We focus on the third-order distortions as they used to prevail over the other distortions

$$
H D 3=\frac{1}{4} \frac{\left|G_{3}\left(j \omega_{1}, j \omega_{1}, j \omega_{1}\right)\right|}{\left|G_{1}\left(j \omega_{1}\right)\right|} A^{2}
$$

where $A$ stands for the amplitude of a sinusoidal signal at the driver input. Using the transfer functions (A.11) and (A.13) derived in Appendix-A, HD3 of the proposed driver can be estimated by

$$
\begin{aligned}
& H D 3_{S F}=\frac{1}{4} \frac{A^{2}}{g_{1 A}+g_{1 B}} \times \\
& \frac{\left(g_{3 A}+g_{3 B}\right)}{\left[1+R_{S}\left(g_{1 A}+g_{1 B}\right)\right]^{3}}-\frac{2 R_{S}\left(g_{2 A}+g_{2 B}\right)^{2}}{\left[1+R_{S}\left(g_{1 A}+g_{1 B}\right)\right]^{4}}
\end{aligned}
$$

Similarly, using (A.14) and (A.16), we can calculate HD3 of the DS-only driver

$$
H D 3_{C S}=\frac{1}{4} \frac{\left(g_{3 A}+g_{3 B}\right)}{g_{1 A}+g_{1 B}} A^{2}
$$

For the bias conditions of $V_{g s A}=1.2 \mathrm{~V}, V_{g s B}=0.75 \mathrm{~V}, V_{d d}=1.2$ $\mathrm{V}, R s=50 \Omega$, and using the same transistor sizes for both circuits $\left((L / W)_{\mathrm{MA}}=(20 / 0.065), \quad(L / W)_{\mathrm{MB}}=(17 / 0.065)\right)$, the following transconductances are achieved: $g_{1 A}=19.6 \mathrm{mS}, g_{1 B}=$ $5.2 \mathrm{mS}, g_{2 A}=1.6 \mathrm{mS}, g_{2 B}=44 \mathrm{mS}, g_{3 A}=-50 \mathrm{mS}, g_{3 B}=158.9$ $\mathrm{mS}$. The comparison of (33) and (34) with these values shows HD3 of the proposed circuit to be by $26.8 \mathrm{~dB}$ better than that of the DS-only circuit. This result is very close to the simulation result shown in Fig. 14. For $300 \mathrm{mV}_{\mathrm{pp}}$ output swing and $50 \Omega$ resistive load, $\mathrm{HD} 3<-80 \mathrm{~dB}$ is attained that corresponds to 13bit DAC resolution.

Within the same bias voltage range also the second-order distortion term can be partly cancelled. Using the Volterra model we have 


$$
H D 2=\frac{1}{2} \frac{\left|G_{2}\left(j \omega_{1}, j \omega_{1}\right)\right|}{\left|G_{1}\left(j \omega_{1}\right)\right|} A
$$

and from (A.11) and (A.12)

$$
H D 2_{S F}=\frac{1}{2} \frac{\left(g_{2 A}+g_{2 B}\right)}{\left(g_{1 A}+g_{1 B}\right)} \frac{1}{\left[1+R_{S}\left(g_{1 A}+g_{1 B}\right)\right]^{2}} A
$$

Similarly, from (A.14) and (A.15)

$$
H D 2_{C S}=\frac{1}{2} \frac{\left(g_{2 A}+g_{2 B}\right)}{g_{1 A}+g_{1 B}} A
$$

By comparison of (36) and (37), the proposed driver shows HD2 by $15 \mathrm{~dB}$ better than its counterpart. This result is also verified by simulations presented in Fig. 15. As the driver is designed as a diffrential circuit a mismatch between its two branches was introduced to model HD2. Also HD3 proves immune to the mismatch.

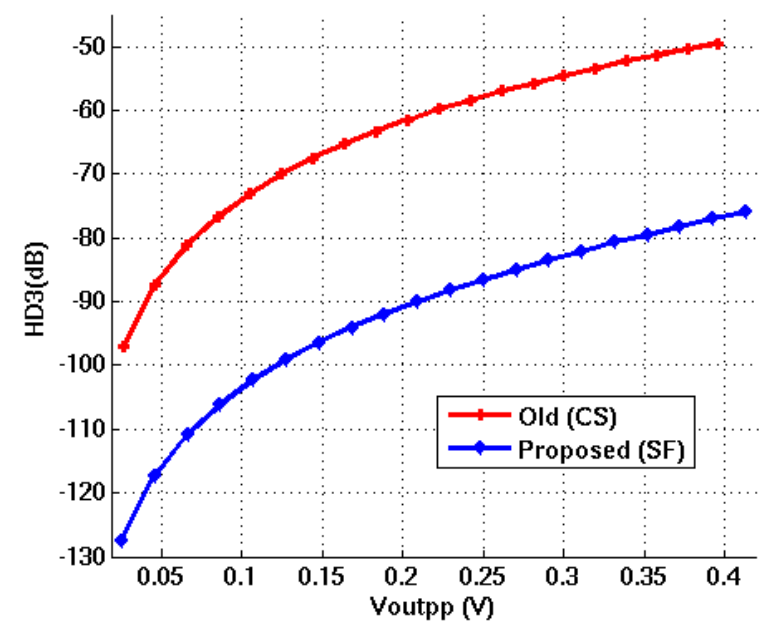

Figure 14. Comparison of conventional DS technique and DS technique combined with resistive feedback.

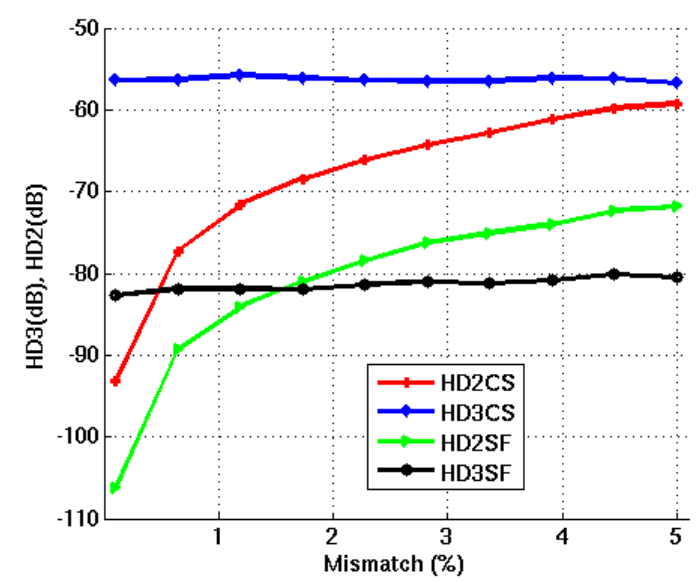

Figure 15. Comparison conventional (CS) and proposed DS technique with resistive load (SF) under mismatch.

For high frequency operation the driver circuit model would be refined as shown in Fig. 16. The parasitic resistances and capacitances $r_{O A}, r_{o B}, C_{d s A}$ and $C_{d s B}$ of the two transistors can be merged as $Z_{\text {out }}=\left(Z_{S}\left\|r_{o A}\right\| r_{o B}\left\|\left(1 / s C_{d s A}\right)\right\|\left(1 / s C_{d s B}\right)\right.$ with $Z_{S}=$ $\left(R_{S} \|\left(1 / s C_{\text {load }}\right)\right.$ and $C_{g s}=C_{g s A}+C_{g s B}$. Since $r_{o A}, r_{o B} \gg R_{S}$ and $C_{\text {load }} \gg C_{d s B}, C_{d s B}$, we can approximate the driver output impedance as $Z_{\text {out }}=\left(R_{S} \|\left(1 / s C_{\text {load }}\right)\right.$.

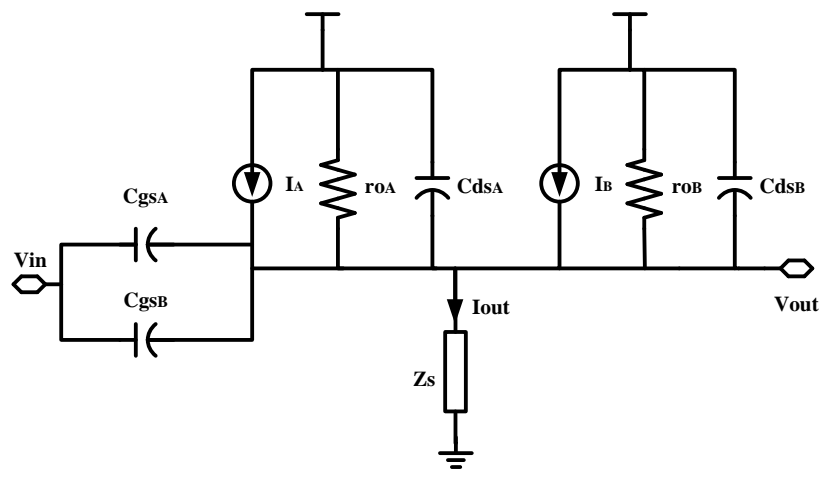

Figure 16. Equivalent circuit of the proposed linear output driver.

Using (32) and (A.24), (A.26) of Appendix-B we can calculate HD3 of the output voltage in the proposed circuit

$$
\begin{aligned}
& H D 3=\frac{A^{2}}{4} \times \frac{1+Z_{\text {OUT }}\left(\omega_{1}\right)\left[g_{1 A}+g_{1 B}+j\left(\omega_{1}\right) C_{g s}\right]}{\mid 1+Z_{\text {OUT }}\left(\omega_{1}+\omega_{2}+\omega_{3}\right)\left[g_{1 A}+g_{1 B}+j\left(\omega_{1}+\omega_{2}+\omega_{3}\right) C_{g s}\right]} \times \\
& \left|\frac{Z_{\text {OUT }}\left(\omega_{1}+\omega_{2}+\omega_{3}\right)\left[\left(g_{3 A}+g_{3 B}\right)\left(1-G_{1}(\omega)\right)^{3}-2\left(g_{2 A}+g_{2 B}\right)\left[G_{2}\left(\omega_{1}+\omega_{2}\right)-\overline{G_{2}\left(\omega_{1}+\omega_{2}\right) G_{1}(\omega)}\right]\right.}{Z_{\text {OUT }}\left(\omega_{1}\right)\left[g_{1 A}+g_{1 B}+j\left(\omega_{1}\right) C_{g s}\right]}\right|
\end{aligned}
$$

HD3 obtained by the Volterra series model (38) and by SpectreRFTM simulations is depicted in Fig. 17 for two $C_{g s}$ values. HD3 tends to degrade with frequency beginning from the low cut-off frequency of $Z_{\text {OUT. }}$. For higher frequencies the ratio $Z_{\text {OUT }}\left(j \omega_{1}, j \omega_{2}, j \omega_{3}\right) / Z_{\text {OUT }}\left(j \omega_{1}\right) \rightarrow 1 / 3$. Also the other numerator to denominator terms tend to settle and this effect is more pronounced for the larger $C_{g s}$ value, which provides better HD3 for higher frequencies at the expense of reduced voltage gain. 


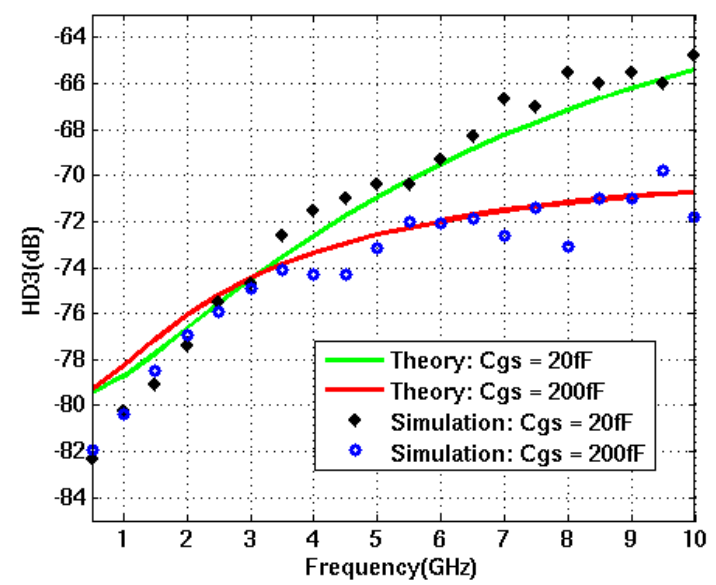

Figure 17. HD3 comparison of Volterra series model (38) and SpectreRF circuit simulations for proposed output driver.

\section{Thermal noise analysis:}

Using the schematic shown in Fig. 13 we can replace the transistors MA and MB by one device with transconductance $g_{m}=g_{m A}+g_{m B}$ and the corresponding input-referred voltage noise PSD of $4 k T \gamma / g_{m}$. By simple calculations the noise by the $R_{S}$ resistor can be found as $4 k T R_{S} /\left(g_{m} R_{S}\right)^{2}$ whereas the total input-referred noise PSD would be

$$
\overline{e_{n}^{2}}=4 k T\left(\frac{\gamma}{g_{m}}+\frac{1}{g_{m}^{2} R_{S}}+\frac{R_{B}}{2}\right)
$$

This can be compared to SC noise (7)

$$
\frac{7 k T}{9 C_{u} f_{S}}=4 k T\left(\frac{\gamma}{g_{m}}+\frac{1}{g_{m}^{2} R_{S}}+\frac{R_{B}}{2}\right)
$$

For example, using $C_{u}=200 \mathrm{fF}, f_{S}=3 \mathrm{GHz}, \gamma=1, g_{m}=40 \mathrm{mS}$, and $R_{S}=50 \Omega$, from (40) we find $R_{B} \approx 600 \Omega$ which can be considered the upper bound of the bias resistance.

\section{Simulation Results}

The circuit has been simulated using Cadence ${ }^{\mathrm{TM}}$ software with RF transistor models ( $65 \mathrm{~nm}$ CMOS). The driver output will be connected to an off-chip load $(50 \Omega)$ and parasitic capacitances caused by wire-bonding cannot be avoided. In simulation we assume that parasitic capacitance equal to $100 \mathrm{fF}$. For signal frequencies up to $5 \mathrm{GHz}$ the gain drop remains within $0.5 \mathrm{~dB}$ shown in Fig. 18.

Using $1.2 \mathrm{~V}$, we distribute the supply current between the main and the auxiliary transistor to be $15.4 \mathrm{~mA}$ (providing $g_{1 \mathrm{~A}}$ $=37.1 \mathrm{mS}$ ) and $1.2 \mathrm{~mA}$ (providing $g_{1 B}=2.9 \mathrm{mS}$ ), respectively. The total current through $R_{s}$ would be $16.6 \mathrm{~mA}$ with the total $g_{m}$ of $40 \mathrm{mS}$. With $R_{s}=65 \Omega$ and $R_{\text {load }}=50 \Omega$, the open-loop gain can be calculated as $\left(R_{\text {load }} \| R_{s}\right) \times\left(g_{1 A}+g_{1 B}\right)=1.13$ which results in the feedback-loop gain of $-5.5 \mathrm{~dB}$.

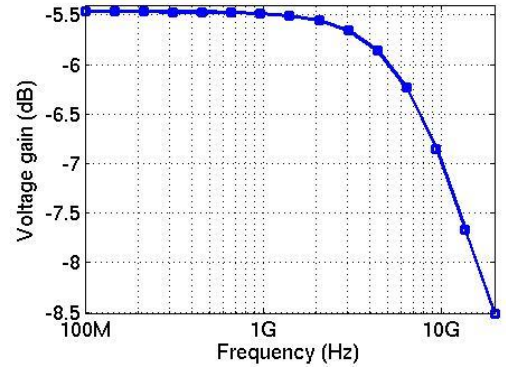

Figure 18. Voltage gain of output driver.

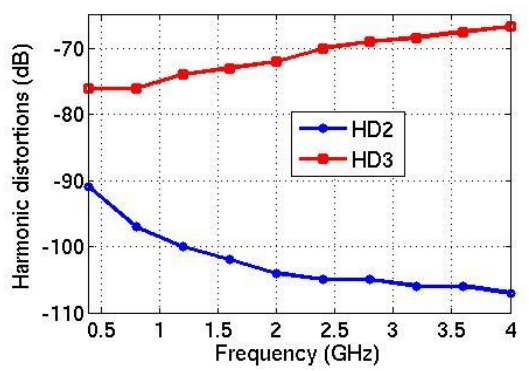

Figure 19. HD3 and HD2 versus frequency.

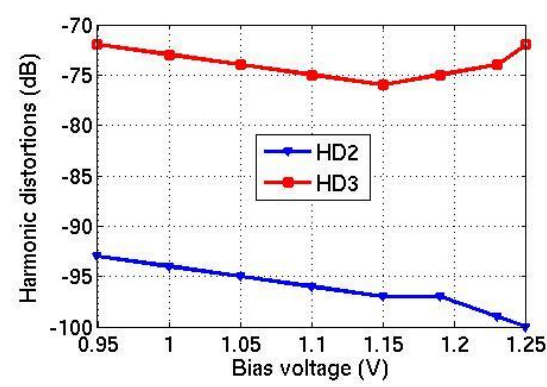

Figure 20. HD3, HD2 versus bias voltage.

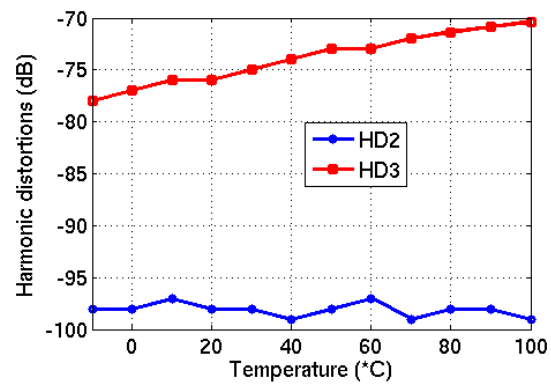

Figure 21. HD3and HD2 versus temperature.

Fig. 18 shows the voltage gain of the driver close to $-5.5 \mathrm{~dB}$ over the band of $0.1-5 \mathrm{GHz}$. It changes only by $0.5 \mathrm{~dB}$ at 5 $\mathrm{GHz}$ and this drop is mainly due to the intentionally added parasitic capacitance $100 \mathrm{fF}$. The corresponding cut-off frequency of the driver is approx. $20 \mathrm{GHz}$. 


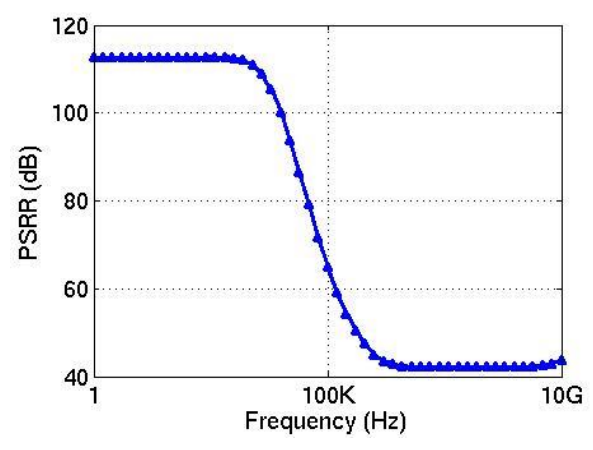

Figure 22. PSRR with 5\% mismatch.

Within the band of $0.5-4 \mathrm{GHz}, \mathrm{HD} 2<-90 \mathrm{~dB}$ and HD3 $<-66$ $\mathrm{dB}$ are attained as shown in Fig. 19. The dependence of HD2 and HD3 on the bias voltage is demonstrated in Fig. 20. HD3 < $-70 \mathrm{~dB}$ and $\mathrm{HD} 2<-90 \mathrm{~dB}$ are achieved with the change of bias voltage from $0.95 \mathrm{~V}$ to $1.25 \mathrm{~V}$. The temperature variation is also verified and shown in Fig. 21. HD2 is not sensitive to temperature whereas HD3 is always less than $-70 \mathrm{~dB}$ in the range of $-10-100{ }^{\circ} \mathrm{C}$.

The power supply rejection ratio (PSRR) is verified for a sinusoidal disturbance imposed on $V_{d d}$ over a wide frequency range as shown in Fig. 22. With intentionally introduced $5 \%$ mismatch into two branches of the differential circuit, PSRR is always greater than $40 \mathrm{~dB}$.

The SC array is designed based on the available design area $\mathrm{W}-\mathrm{C}_{\mathrm{u}}$ for given resolution and signal bandwidth. For 7- and 8bit applications with $3 \mathrm{GHz}$ clock, according to Fig. 9 and Fig. 10 we choose $C_{u}=200 \mathrm{fF}$ and the total switch size $W=64 \mu \mathrm{m}$ (16 $\mu \mathrm{m}$ for NMOS and $48 \mu \mathrm{m}$ for PMOS) with $L=60 \mathrm{~nm}$. The necessary dummies of a similar size are applied.

The simulation results of the complete SC DAC for 7- and 8 -bit resolution are shown in Fig. 23 for OSR $=1.1$. For frequencies above $3.5 \mathrm{GHz}$ the SFDR drops due to violation of the settling time. The attained SFDR is not affected by the output driver for its high linearity and bandwidth.

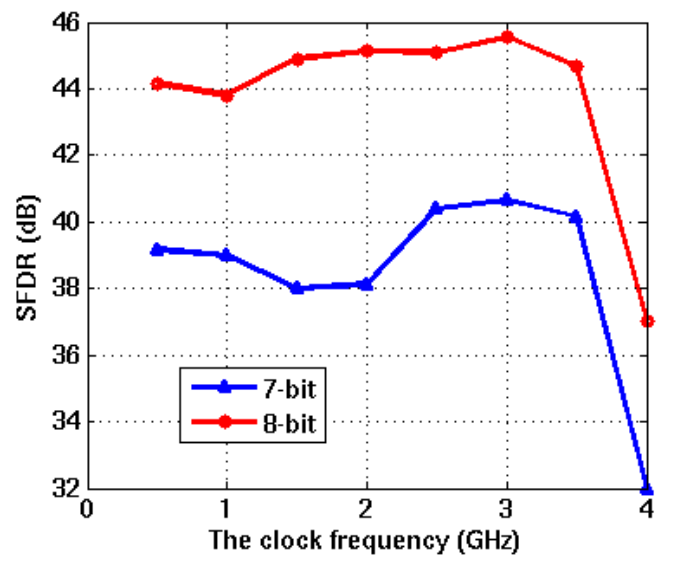

Figure 23. SFDR against clock frequency for 7-bit and 8-bit $(\mathrm{OSR}=1.1)$.

For 6-bit resolution with $12 \mathrm{GHz}$ clock frequency the unit capacitance value must be largely reduced according to the available design area shown in Fig. 11. In this case we choose $C_{u}=30 \mathrm{fF}$ while using the same size of switches $(W=64 \mu \mathrm{m})$.
The simulation results of the complete SC DAC with 6-bit resolution are shown in Fig. 24 for OSR $=1.1$. The attained SFDR approaches $34 \mathrm{~dB}$ but for clock frequencies below $8 \mathrm{GHz}$ it is largely deteriorated due to leakage effects caused by too low time constant $R_{o f f} C_{u}$. In fact, this could be seen as the lower and upper bounds on $C_{u}$, shown in Fig. 11, to go up for lower clock frequencies while the actual operating point $\left(C_{u}, W\right)$ remains unchanged and ultimately it falls out of the design area.

The DAC power consumption is mostly due to the output driver and the digital clock part $\left(75 \mathrm{~mW} @ f_{s}=3 \mathrm{GHz}\right)$. The pipeline SC array consumes much less power $\left(15 \mathrm{~mW} @ f_{s}=\right.$ $3 \mathrm{GHz}$ ) since its total capacitance is relatively low and the operation is based on charge redistribution. The summary of the SC DAC performance is presented in Table I.

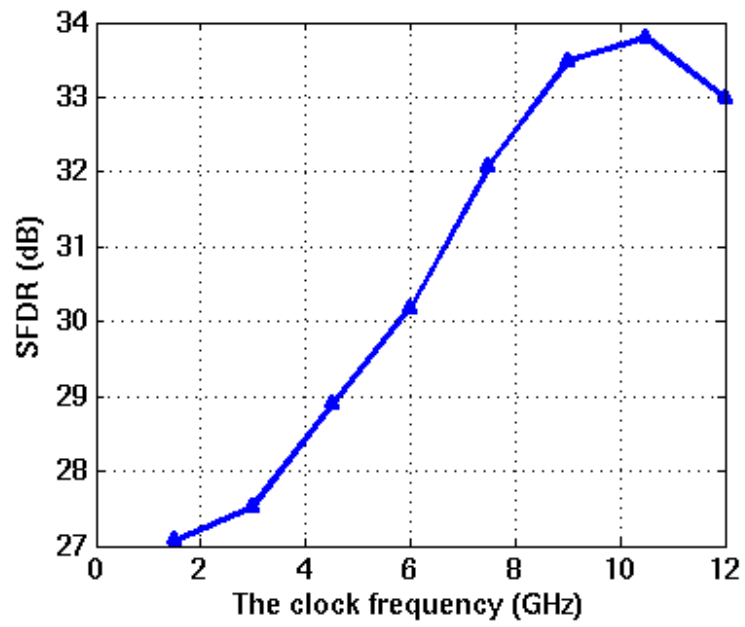

Figure 24. SFDR against clock frequency (OSR $=1.1)$ for $n=6$.

TABLE I. SUMMARY OF SC DAC PERFORMANCE.

\begin{tabular}{c|c|c|c} 
Technology & \multicolumn{3}{|c}{$65 \mathrm{~nm}$ CMOS } \\
\hline Supply voltage & \multicolumn{3}{|c}{$1.2 \mathrm{~V}$} \\
\hline Power consumption & \multicolumn{3}{|c}{$90 \mathrm{~mW} @ f_{s}=3 \mathrm{GHz}$} \\
\hline Resolution & 8 -bit & 7 -bit & 6-bit \\
\hline SFDR [dB] & 45 & 40 & 33 \\
\hline$f_{s}[\mathrm{GHz}]$ & 3 & 3 & 12 \\
\hline$f_{\text {in }}[\mathrm{GHz}]$ & 1.36 & 1.36 & 5.5
\end{tabular}

There are 5 definitions of figure of merit (FOM) in [2, 25] but only two of them are generic i.e. suitable for any kind of DAC type. They are FOM2 and FOM3

$$
\begin{aligned}
& \text { FOM } 2=\frac{2^{N} B W_{N}}{P_{\text {total }}} \\
& \text { FOM } 3=\frac{V_{\text {swing }}}{P_{\text {total }}} B W 10^{\frac{S F D R}{20}}
\end{aligned}
$$

where $N$ is the number of bits, $B W$ (or $B W_{N}$ ) is the signal bandwidth, $P_{\text {total }}$ is the total power consumption, whereas 
$V_{\text {swing }}$ and SFDR stand for the differential output swing and spurious free dynamic range of DACs, respectively.
Table II gives performance comparison of the presented design and other state-of-the-art publications.

TABLE II. DAC PERFORMANCE SUMMARY AND COMPARISON

\begin{tabular}{|c|c|c|c|c|c|c|c|c|c|c|c|c|}
\hline Ref & $\begin{array}{c}\text { DAC } \\
\text { type }\end{array}$ & $\begin{array}{c}\text { Technology } \\
{[\mathrm{nm}]}\end{array}$ & $\begin{array}{c}\text { Supply } \\
\text { [V] }\end{array}$ & $\begin{array}{c}\text { Fclk } \\
{[\mathrm{GHz}]}\end{array}$ & $\begin{array}{c}\text { BW } \\
{[\mathbf{G H z}]}\end{array}$ & $\begin{array}{c}\text { SFDR } \\
{[\mathrm{dB}]}\end{array}$ & $\begin{array}{l}\text { IM3 } \\
\text { [dB] }\end{array}$ & $\begin{array}{c}\text { Swing } \\
{[\text { Vppd] }}\end{array}$ & $\begin{array}{l}\text { Power } \\
{[\mathrm{mW}]}\end{array}$ & Bits & $\begin{array}{c}\text { FOM2 } \\
{\left[10^{12}\right]} \\
\end{array}$ & $\begin{array}{c}\text { FOM3 } \\
{\left[10^{12}\right]}\end{array}$ \\
\hline $\begin{array}{c}1] \\
\text { JSSC } 01\end{array}$ & $\begin{array}{l}\text { Current } \\
\text { steering }\end{array}$ & 350 & $3 / 1.9$ & 1 & 0.49 & 61.2 & - & 1.6 & 110 & 10 & 4.6 & 8.2 \\
\hline $\begin{array}{c}{[2]} \\
\text { JSSC } 08 \\
\end{array}$ & $\begin{array}{l}\text { Current } \\
\text { steering }\end{array}$ & 130 & 1.2 & 3 & 1.426 & 36.2 & -39.5 & 0.4 & 29 & 6 & 3.2 & 1.3 \\
\hline $\begin{array}{c}{[27]} \\
\text { JSSC } 08\end{array}$ & $\begin{array}{l}\text { Current } \\
\text { steering }\end{array}$ & 90 & $1.8 / 1$ & 12 & 1.5 & 35 & - & 1.6 & 190 & 8 & 2.02 & 0.71 \\
\hline $\begin{array}{c}3] \\
\text { JSSC } 09\end{array}$ & $\begin{array}{l}\text { Current } \\
\text { steering }\end{array}$ & 65 & 2.5 & 2.9 & 0.55 & 60 & -60 & 2.5 & 188 & 12 & 12 & 7.3 \\
\hline $\begin{array}{c}{[26]} \\
\text { ISSC } 11 \\
\end{array}$ & $\begin{array}{l}\text { Current } \\
\text { steering }\end{array}$ & 65 & $2.5 / 1.1$ & 56 & 26.9 & 30 & - & 0.6 & 750 & 6 & 2.3 & 0.7 \\
\hline $\begin{array}{c}{[28]} \\
\text { TCAS13 }\end{array}$ & $\Delta \Sigma$ & 65 & $1.2 / 1$ & 8 & 0.2 & $\begin{array}{c}26 \\
\text { SNDR }\end{array}$ & -57 & 0.3 & 68 & 12 & 12 & - \\
\hline $\begin{array}{c}5] \\
\text { JSSC } 04\end{array}$ & $\mathrm{SC}$ & 500 & 3.3 & 0.2 & 0.034 & 70 & - & 2 & 693 & 10 & 0.05 & 0.3 \\
\hline $\begin{array}{c}{[6]} \\
\text { ASSC10 }\end{array}$ & $\mathrm{SC}$ & 90 & $2.5 / 1.2$ & 0.8 & 0.4 & 53 & - & 2.9 & 103 & 12 & 15.9 & 5 \\
\hline $\begin{array}{c}\text { This } \\
\text { work }^{(a)}\end{array}$ & SC & 65 & 1.2 & $3 / 12$ & $1.38 / 5.5$ & $45 / 33$ & $\begin{array}{l}-45 / \\
-33 \\
\end{array}$ & $0.3 / 0.3$ & $90 / 130$ & $8 / 6$ & $3.9 / 2.7$ & $1.4 / 1$ \\
\hline
\end{tabular}

(a) Simulation

\section{CONCLUSION}

In this paper we have presented a pipeline SC DAC design in $65 \mathrm{~nm}$ CMOS for high-speed applications. With $300 \mathrm{mV}_{\mathrm{pp}}$ output voltage range and $\mathrm{GHz}$ bandwidth the $\mathrm{DAC}$ is well suited for the contemporary wideband wireless transmitters. Having designed a highly linear output driver we have found the SC array to limit the DAC performance mostly due to the settling and clock feed-through effect rather than $\mathrm{kT} / \mathrm{C}$ noise and capacitor mismatch. By precise cancelation of the clock feed-through the SFDR was shown to follow well the resolution up to 8 bits for $\mathrm{GHz}$ signal bandwidth ( $45 \mathrm{~dB}$ for $n=8$ and $B W=1.36 \mathrm{GHz}$ or $33 \mathrm{~dB}$ for $n=6$ and $B W=$ $5.5 \mathrm{GHz}$ ) without extra means of correction. Thereby the tradeoff between DAC bandwidth and resolution accompanied by SFDR has been demonstrated. Higher SFDR can be attained by reducing the clock frequency for increased number of bits and redesigning the $\mathrm{SC}$ array accordingly. However, from analysis of the available $\left(C_{u}, W\right)$ design area we find increasing the resolution from 8 to 9 bits with the corresponding improvement of SFDR, feasible only for clock frequency reduced below $0.5 \mathrm{GHz}$. In such a case the data rate can be elevated by employing the interleaving technique [6] or using a newer CMOS technology.

The main contribution of this paper is showing an alternative way to design high-speed DACs beside currentsteering technique which has been widely used. The existing SC DACs have been shown competitive mostly in terms of power consumption for no static current in their SC circuits. Even more advantages can be attained in deep submicron technologies. Using $28 \mathrm{~nm}$ FD SOI for example, the SC circuits with their capacitances and on-resistance switches can achieve better matching than their current-steering counterparts where the transistors operate in saturation region. The output impedances of current-cells decrease with scaling down due to lower supply, resulting also in more distortion at the DAC output, whereas SC arrays do not have this problem.

\section{APPENDIX A \\ DERIVATION OF THE VOLTERRA OPERATORS FOR THE PROPOSED DRIVER AT LOW FREQUENCY}

For the circuit shown in Fig. 13(a) the respective currents and voltages can be expressed as

$$
\begin{gathered}
i_{A}=g_{1 A} v_{g s}+g_{2 A} v_{g s}{ }^{2}+g_{3 A} v_{g s}{ }^{3} \\
i_{B}=g_{1 B} v_{g s}+g_{2 B} v_{g s}{ }^{2}+g_{3 B} v_{g s}{ }^{3} \\
i_{\text {OUT }}=i_{A}+i_{B} \\
v_{g s}=v_{\dot{i} r}-v_{\text {OUT }} \\
v_{\text {OUT }}=R_{S} i_{\text {OUT }}
\end{gathered}
$$

Where $g_{i A}$ and $g_{i B}$ are the $i^{t h}$-order coefficients of MA and MB, accordingly, obtained by taking the derivative of the drain dc current $I_{D S}$ with respect to the gate-source voltage $V_{G S}$ at the dc bias point

$$
\begin{gathered}
g_{1 A}=\frac{\partial I_{D S A}}{\partial V_{G S A}}, g_{1 B}=\frac{\partial I_{D S B}}{\partial V_{G S B}}, g_{1}=g_{1 A}+g_{1 B} \\
g_{2 A}=\frac{1}{2 !} \frac{\partial^{2} I_{D S A}}{\partial V_{G S A}^{2}}, g_{2 B}=\frac{1}{2 !} \frac{\partial^{2} I_{D S B}}{\partial V_{G S B}^{2}}, g_{2}=g_{2 A}+g_{2 B}
\end{gathered}
$$




$$
g_{3 A}=\frac{1}{3 !} \frac{\partial^{3} I_{D S A}}{\partial V_{G S A}^{3}}, g_{3 B}=\frac{1}{3 !} \frac{\partial^{3} I_{D S B}}{\partial V_{G S B}^{3}}, g_{3}=g_{3 A}+g_{3 B}
$$

Applying the Volterra series to the output voltage

$$
v_{\text {OUT }}=G_{1} \circ v_{i n}+G_{2} \circ v_{i n}^{2}+G_{3} \circ v_{i n}^{3}
$$

Substituting (A.1-A.4) into (A.5), we have

$$
\begin{aligned}
& v_{\text {OUT }}=R_{S}\left(g_{1 A}+g_{1 B}\right)\left(v_{i n}-v_{\text {OUT }}\right) \\
& +R_{S}\left(g_{2 A}+g_{2 B}\right)\left(v_{i n}-v_{\text {OUT }}\right)^{2}+R_{S}\left(g_{3 A}+g_{3 B}\right)\left(v_{i n}-v_{\text {OUT }}\right)^{3}
\end{aligned}
$$

Then from (A.9) and (A.10) the functions $G_{1}, G_{2}$, and $G_{3}$ are obtained respectively

$$
\begin{gathered}
G_{1}=\frac{R_{S}\left(g_{1 A}+g_{1 B}\right)}{1+R_{S}\left(g_{1 A}+g_{1 B}\right)} \\
G_{2}=\frac{R_{S}\left(g_{2 A}+g_{2 B}\right)}{\left[1+R_{S}\left(g_{1 A}+g_{1 B}\right)\right]^{3}} \\
G_{3}=\frac{R_{S}\left(g_{3 A}+g_{3 B}\right)}{\left[1+R_{S}\left(g_{1 A}+g_{1 B}\right)\right]^{4}}-\frac{2\left[R_{S}\left(g_{2 A}+g_{2 B}\right)\right]^{2}}{\left[1+R_{S}\left(g_{1 A}+g_{1 B}\right)\right]^{5}}
\end{gathered}
$$

In a similar way, for the DS-only driver we find

$$
\begin{aligned}
& G_{1}=R_{S}\left(g_{1 A}+g_{1 B}\right) \\
& G_{2}=R_{S}\left(g_{2 A}+g_{2 B}\right) \\
& G_{3}=R_{S}\left(g_{3 A}+g_{3 B}\right)
\end{aligned}
$$

\section{APPENDIX B}

\section{DERIVATION OF THE VOLTERRA OPERATORS FOR THE} PROPOSED DRIVER AT HIGH FREQUENCY

For the circuit of Fig. 16 the current and voltage equations follow

$$
\begin{gathered}
i_{A}=g_{1 A} v_{g s}+g_{2 A} v_{g s}^{2}+g_{3 A} v_{g s}^{3} \\
i_{B}=g_{1 B} v_{g s}+g_{2 B} v_{g s}{ }^{2}+g_{3 B} v_{g s}{ }^{3} \\
i_{\text {OUT }}=i_{A}+i_{B}+\left(v_{i t}-v_{\text {OUT }}\right) s C_{g s} \\
v_{g s}=v_{i r}-v_{\text {OUT }} \\
v_{\text {OUT }}=Z_{\text {OUT }} i_{\text {OUT }}
\end{gathered}
$$

For the Volterra series applied to the output voltage

$$
v_{\text {oUT }}=G_{1} \circ v_{\dot{i n}}+G_{2} \circ v_{\dot{i n}}^{2}+G_{3} \circ v_{\dot{i n}}^{3}
$$

Substituting (A.17-A.20) into (A.21), we have

$$
\begin{aligned}
& v_{\text {OUT }}=Z_{\text {OUT }}\left(g_{1 A}+g_{1 B}+s C_{g s}\right)\left(v_{i n}-v_{\text {OUT }}\right) \\
& +Z_{\text {OUT }}\left(g_{2 A}+g_{2 B}\right)\left(v_{i n}-v_{\text {OUT }}\right)^{2} \\
& +Z_{\text {OUT }}\left(g_{3 A}+g_{3 B}\right)\left(v_{i n}-v_{\text {OUT }}\right)^{3}
\end{aligned}
$$

Next, using (A.22) and (A.23) the Volterra transfer functions $G_{1}, G_{2}$, and $G_{3}$ are obtained

$$
\begin{aligned}
& G_{1}(\omega)=\frac{Z_{\text {OUT }}(\omega)\left(g_{1 A}+g_{1 B}+j \omega C_{g s}\right)}{1+Z_{\text {OUT }}(\omega)\left(g_{1 A}+g_{1 B}+j \omega C_{g s}\right)} \\
& G_{2}\left(\omega_{1}+\omega_{2}\right)=\frac{Z_{\text {OUT }}\left(\omega_{1}+\omega_{2}\right)\left(g_{2 A}+g_{2 B}\right)\left(1-G_{1}(\omega)\right)^{2}}{\left[1+Z_{\text {OUT }}\left(\omega_{1}+\omega_{2}\right)\left(g_{1 A}+g_{1 B}+j\left(\omega_{1}+\omega_{2}\right) C_{g s}\right)\right]^{3}} \\
& G_{3}\left(\omega_{1}+\omega_{2}+\omega_{3}\right)= \\
& \frac{Z_{\text {OUT }}\left(\omega_{1}+\omega_{2}+\omega_{3}\right)\left(g_{3 A}+g_{3 B}\right)\left(1-G_{1}(\omega)\right)^{3}}{1+Z_{\text {OUT }}\left(\omega_{1}+\omega_{2}+\omega_{3}\right)\left[g_{1 A}+g_{1 B}+j\left(\omega_{1}+\omega_{2}+\omega_{3}\right) C_{g s}\right]} \\
& -\frac{2 Z_{\text {OUT }}\left(\omega_{1}+\omega_{2}+\omega_{3}\right)\left(g_{2 A}+g_{2 B}\right)\left[G_{2}\left(\omega_{1}+\omega_{2}\right)-\overline{G_{2}\left(\omega_{1}+\omega_{2}\right) G_{1}(\omega)}\right]}{1+Z_{\text {OUT }}\left(\omega_{1}+\omega_{2}+\omega_{3}\right)\left[g_{1 A}+g_{1 B}+j\left(\omega_{1}+\omega_{2}+\omega_{3}\right) C_{g s}\right]}
\end{aligned}
$$

where

$$
\begin{aligned}
& \overline{G_{2}\left(\omega_{1}+\omega_{2}\right) G_{1}(\omega)}= \\
& \frac{\left[G_{2}\left(\omega_{1}+\omega_{2}\right) G_{1}\left(\omega_{3}\right)+G_{2}\left(\omega_{1}+\omega_{3}\right) G_{1}\left(\omega_{2}\right)+G_{2}\left(\omega_{3}+\omega_{2}\right) G_{1}\left(\omega_{1}\right)\right]}{3}
\end{aligned}
$$

\section{APPENDIX C \\ SIMULATION RESULTS FROM 28 NM CMOS FD SOI TENOLOGY}

A more advanced technology such as $28 \mathrm{~nm}$ CMOS FD SOI is used to show advantages of SC pipeline DAC due to scaling and other features available in this case. Using this technology the design areas demonstrated in Section III are becoming larger for the same clock frequency and resolution. This can be seen from comparison of the transistor width range shown in Fig. 8 and Fig. 25, where the latter shows $\left[W_{\min }, W_{\max }\right]=[63,3000] \mu \mathrm{m}$. However, the real advantage is that higher frequencies and resolutions can be attained with this technology.

In FD SOI technology the oxide capacitance $C_{o x}$ is much smaller than in standard bulk CMOS so $C_{g}(W)$ is smaller and the channel length $L=30 \mathrm{~nm}$ results in less $R_{\text {on }}$ as well. That is why the upper bounds for $C_{u}$ are higher than the ones obtained with $65 \mathrm{~nm}$ technology. A smaller value of $C_{o x}$ also results in smaller $C_{p}(W)$ in (20) which makes the $C_{u}$ lower bound (due to feed-through effect) more relaxed as well.

In particular, at $3 \mathrm{GHz}$ clock frequency as demonstrated in Fig. 26 it is possible to design 11-bit SC DAC using $\left(W, C_{u}\right)=$ (125 $\mu \mathrm{m}, 500 \mathrm{fF}$ ) with a sufficient design area around to guarantee circuit robustness. The value of unit capacitance is larger than required by $\mathrm{kT} / \mathrm{C}$ noise condition (14) $C_{u}>50 \mathrm{fF}$ for $n=11$. Observe that the lower $C_{u}$ bound is, in fact, dictated by the feedthrough condition (20) (green line) and not by $R_{\text {off }}$ in (31). It also shows that 12-bit DAC at $3 \mathrm{GHz}$ is not feasible (green dotted line) unless the demands for accuracy are relaxed to one LSB.

Additionally, in Fig. 27 we show the speed capability of a 9bit SC DAC that with a tight design area demonstrates a theoretical data rate of $135 \mathrm{Gbps}$. 


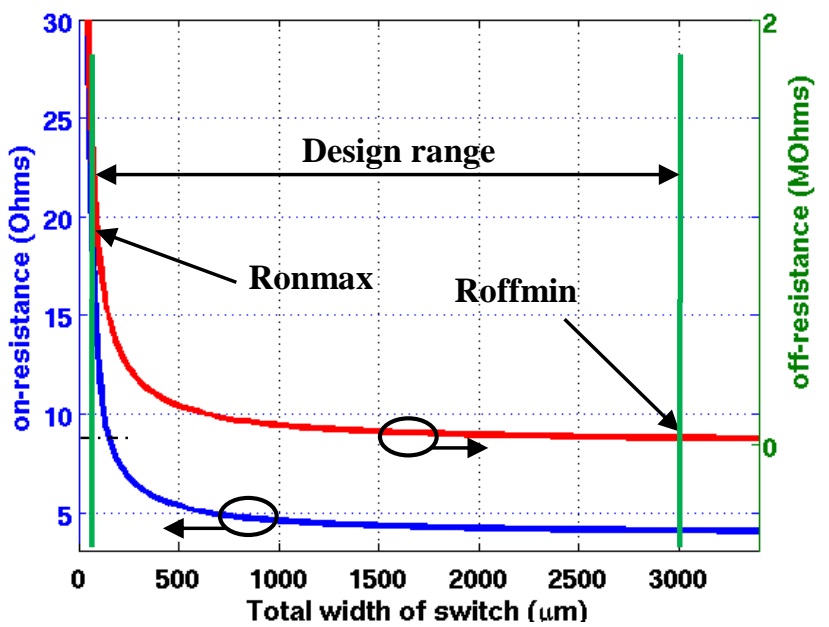

Figure 25. Off-resistance and on-resistance of T-gate switch vs. width in $28 \mathrm{~nm}$ CMOS FD SOI with $L=30 \mathrm{~nm}\left(W_{p} / W_{n}=3\right)$.

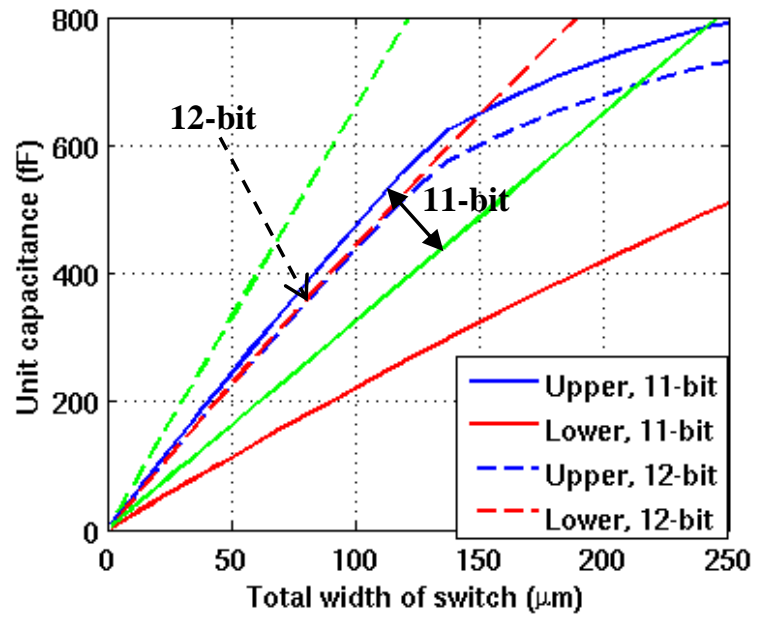

Figure 26. Design area $\mathrm{W}_{-} \mathrm{C}_{\mathrm{u}}$ for $28 \mathrm{~nm}$ CMOS FD SOI, 11-bit and 12-bit SC DAC assuming half LSB accuracy, $f_{S}=3 \mathrm{GHz}, K_{d i f}=20 \%$ and $K_{\text {comp }}=1 \%$.

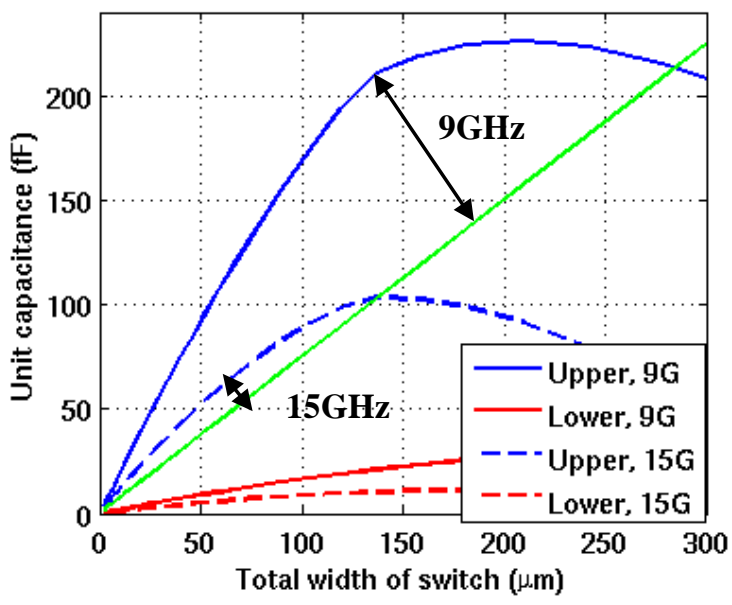

Figure 27. Design area $\mathrm{W}_{-} \mathrm{C}_{\mathrm{u}}$ for 28nm CMOS FD SOI, 9-bit SC DAC with different clock frequencies $\left(f_{s}\right), K_{\text {dif }}=20 \%, K_{\text {comp }}=1 \%$.

\section{REFERENCES}

[1] V. D. Bosch, et al.,"A 10-bit 1-GSample/s Nyquist current-steering CMOS D/A converter," IEEE J. Solid-State Circuits, vol. 36, pp. 315324, Mar. 2001.

[2] X. Wu, et al.,"A 130nm CMOS 6-bit Full Nyquist 3 GS/s DAC," IEEE J. Solid-State Circuits, vol. 43, pp. 2396-2403, Nov. 2008.

[3] C. H. Lin, et al.,"A 12 bit 2.9 GS/s DAC with IM3 < -60 dBc Beyond 1 GHz in 65nm CMOS," IEEE J. Solid-State Circuits, vol. 44, pp. 32853293, Dec. 2009.

[4] S. Spiridon, et al.,"A $375 \mathrm{~mW}$ Multimode DAC-Based Transmitter With $2.2 \mathrm{GHz}$ Signal Bandwidth and In-band IM3 $<-58 \mathrm{dBc}$ in $40 \mathrm{~nm}$ CMOS," IEEE J. S.S. Circuits, vol. 48, pp. 1595-1604, July. 2013.

[5] G. Manganaro, et al.,"A Dual 10-b 200-MSPS Pipeline D/A Converter With DLL-Based Clock Synthesizer," IEEE J. Solid-State Circuits, vol. 39, pp. 1829-1838, Nov. 2004.

[6] C. Daigle, et al.,"A 12-bit 800-MS/s Switched-Capacitor DAC with Open-loop Output Driver and Digital Predistortion," IEEE Asian SolidState Circuits Conference, pp. 1-4, Nov. 2010.

[7] D. A. Mercer,"Low Power Approaches to High Speed CMOS Currentsteering DACs," CICC, pp. 153-160, Sept. 2006.

[8] J. L. Dawson, "Feedback Linearization of RF Power Amplifiers," $P h D$ Thesis, Stanford, US, August 2003.

[9] A. Afsahi, et al.,"Linearized Dual-Band Power Amplifer with Integrated Baluns in 65nm CMOS for a 2x2 802.11n MIMO WLAN SoC," IEEE J. Solid-State Circuits, vol. 45, pp. 995-966, May. 2010.

[10] H. Hedayati, et al.,"A 2-GHz Highly Linear Efficient Dual-Mode BiCMOS Power Amplifier Using a Reconfigurable Matching Network," IEEE J. Solid-State Circuits, vol. 47, pp. 2385-2404, Oct. 2012.

[11] Q. Liu, et al.,"A high efficiency and high linearity power amplifier utilizing post-linearization technique for $5.8 \mathrm{GHz}$ DSRC applications," PAWR, pp. 45-48, Jan. 2011.

[12] T. W. Kim, et al.,"Highly linear reciever front-end adopting MOSFET transconductance linearization by multiple gated transistors," IEEE $J$. Solid-State Circuits, vol. 39, pp. 223-229, Jan. 2004.

[13] C. Xin, et al.,"A linearization technique for RF low noise amplifier," ISCAS, pp. 313-316, May. 2004.

[14] H. Zhang, E.Sanchez-Sinencio " Linearization Techniques for CMOS Low Noise Amplifiers: A Tutorial", Transactions on Circuit and Systems, vol. 58, no. 1, January 2011.

[15] Q-T. Duong and J. Dąbrowski, "Low Noise Transconductance Amplifier Design for CT Sigma Delta WB Frontend”, ECCTD, pp. 825-828, 2011.

[16] Y. Ding, et al.,"A Highly-efficiency CMOS +22-dBm Linear Power Amplifier," IEEE J. Solid-State Circuits, vol. 39, pp. 223-229, Jan. 2004.

[17] A. I. Galal, et al.,"Comparision between bipolar and NMOS transistors in linearization technique at $5 \mathrm{GHz}$ low noise amplifier," $A P M C$, pp. 1-4, Dec. 2008.

[18] F. J. Wang, et al.,“A quasipassive CMOS pipeline D/A converter," IEEE J. Solid-State Circuits, vol. 24, pp. 1752-1755, Dec. 1989.

[19] R. E. Suarez, et al.,"All-MOS Charge Redistribution Analog-to-Digital Conversion Techniques-Part II," IEEE J. Solid-State Circuits, vol. 10, pp. 379-385, Dec. 1975.

[20] R. Gregorian, G. Temes, "Analog MOS Integrated Circuits", Wiley, 1986

[21] T. Wakimoto, et al.,"Statistical Analysis on the Effect of Capacitance Mismatch in a High-resolution Successive-Approximation ADC," IEEJ Transactions on electrical and electronic engineering, vol. 6, pp. s89s93, 2001.

[22] Q-T. Duong, J. Dąbrowski, A. Alvandpour, "Highly linear open loop output driver for high speed capacitive DACs", Norchip2013, pp. 1-4, 2013

[23] B. H. Leung, VLSI for Wireless Communication. Englewood Cliffs, NJ: Prentice-Hall, 2002.

[24] R. van de Plassche, CMOS Integrated Analog-to-Digital and Digital-toAnalog Converters. $2^{\text {nd }}$ Edition, Springer, 2007. 
[25] P. Palmers, et al." "A 10-Bit 1.6-GS/s 27-mW Current-Steering D/A Converter With 550-MHz 54-dB SFDR Bandwidth in 130-nm CMOS," IEEE TCASI, vol. 57, pp. 2870-2879, Nov. 2010.

[26] M. G. Yuriy, et al.,"A 56GS/s 6b DAC in 65nm CMOS with 256x6b Memory," ISSC, vol. 10, pp. 194-195, Dec. 2011.

[27] S. Jafar, et al.,"A 12-GS/s Phase-Calibrated CMOS Digital-to-Analog for Backplane Communication," IEEE J. Solid-State Circuits, vol. 43, pp. 1207-1216, May. 2008.

[28] A. Bhide, et al." " An 8-GS/s 200-MHz Bandwidth 68-mW $\Delta \Sigma$ DAC in 65-nm CMOS,” IEEE TCAS II, vol. 60, pp. 387-391, July. 2013. 